\title{
Stimulus features that elicit activity in object-vector cells
}

Sebastian O. Andersson, Edvard I. Moser and May-Britt Moser

Kavli Institute for Systems Neuroscience and Centre for Neural Computation, Norwegian University of Science and Technology (NTNU), Trondheim, Norway

Corresponding authors: Sebastian O. Andersson: sebastian.o.andersson@ntnu.no; May-Britt Moser: may-britt.moser@ntnu.no

Editorial correspondence: edvard.moser@ntnu.no 


\section{Abstract}

Object-vector (OV) cells are cells in the medial entorhinal cortex (MEC) that track an animal's distance and direction to objects in the environment. Their firing fields are defined by vectorial relationships to free-standing 3-dimensional (3D) objects of a variety of identities and shapes. However, the natural world contains a panorama of objects, ranging from discrete 3D items to flat two-dimensional (2D) surfaces, and it remains unclear what are the most fundamental features of objects that drive vectorial responses. Here we address this question by systematically changing features of experimental objects. Using an algorithm that robustly identifies OV firing fields, we show that the cells respond to a variety of $2 \mathrm{D}$ surfaces, with visual contrast as the most basic visual feature to elicit neural responses. The findings suggest that OV cells use plain visual features as vectorial anchoring points, allowing vector-guided navigation to proceed in environments with few free-standing landmarks.

\section{Introduction}

MEC and the adjacent pre- and parasubiculum are critical components of the neural representation of space $^{1,2}$, hosting cell types that dynamically signal the animal's position ${ }^{3-5}$, head direction ${ }^{5-7}$, speed ${ }^{8}$ and proximity to borders ${ }^{5,9,10}$. These cells coexist and interact with a wide range of allocentrically tuned cell types in neighbouring hippocampal-parahippocampal regions, such as place cells ${ }^{11,12}$, boundaryvector cells ${ }^{13-15}$ and landmark-controlled cells ${ }^{16-18}$, as well as various cells that encode position in egocentric coordinates relative to the animal's body axis ${ }^{19-22}$. Collectively, this network of space-coding neurons is thought to enable animals to navigate the world.

Recently, we reported the existence of yet another cell type in MEC: object-vector (OV) cells, which signal the animal's distance and direction to discrete objects in the environment ${ }^{23}$. The large number of OV cells in MEC, on par with the number of grid cells, suggest that tracking of the animal's position in an object-centred space is a major form of spatial representation in $\mathrm{MEC}^{23}$, just as in the wider hippocampal formation ${ }^{13-15}$. OV cells respond to an impressive array of objects: from small to large, narrow to wide, and short to tall, although they are inclined to respond to taller objects ${ }^{23}$. In addition, the cells fire independently of the object's shape, colour, or familiarity. While the stimulus space of objects that OV cells can produce vectors to must be gigantic, the lower bound of this stimulus space is unknown: what are the simplest object features that can elicit activity in OV cells? Here we address this question by parametrically stripping objects of some of their qualities while measuring responses of OV cells. We ask first whether OV cells respond only to free-standing 3D objects by converting in a step-wise manner an object from $3 \mathrm{D}$ to $2 \mathrm{D}$ and testing responses in OV cells. After finding that $2 \mathrm{D}$ surfaces on the wall of the recording environment are sufficient to elicit OV firing, we show that a simple visual contrast on the wall is sufficient to elevate activity.

\section{Results}

\section{Identification of OV cells and OV fields}

To identify the most fundamental features that elicit activity in OV cells, we implanted 7 mice with tetrodes in the MEC (Supplementary Fig. 1), where OV cells are numerous ${ }^{23}$. As in earlier work ${ }^{23}$, we performed three trials to identify OV cells: an 'Empty Box' trial, an 'Object' trial, and a 'Moved Object' trial (Fig. 1a). In the latter pair of trials, the object was a $40 \times 8 \times 8 \mathrm{~cm}$ (height $\times$ base area) multi-colour Duplo tower, known to produce clear OV responses ${ }^{8}$ (Supplementary Fig. 2 ). OV cells were identified by an 'OV score', equivalent to the Pearson correlation between object-centered pairs of rate maps from the 'Object' and 'Moved Object' trials. These rate maps show firing rate as a function of allocentric angle and distance (Fig. 1b). When the cell's firing depends on the animal's position relative to the object, and not on the animal's absolute position, this correlation should be high. 
As in earlier work ${ }^{8}$, cells had to pass multiple criteria to count as OV cells. First, we required the appearance of one or more new fields in the 'Object' trial compared to the 'Empty Box' trial. For each cell passing this criterion, we generated a shuffled spike-time distribution by randomly shifting the cell's spike timestamps along the animal's trajectory $(n=200$ permutations per cell). Cells were defined as OV cells if (i) they had spatial information contents exceeding the 99th percentile of their own shuffling distribution (Fig. 1c), (ii) OV scores exceeding the 99th percentile of their own shuffling distribution (Fig. 1d), and (iii) the firing fields were displaced $>4 \mathrm{~cm}$ away from the object centre. In total, 67 cells passed the triplet of criteria including the spatial information criterion (Fig. 1e) and the OV score criterion (Fig. 1f) and were classified as OV cells.

Since the aim of the present study is to identify the basic features of objects that produce activity in OV cells, we expected some of the more primitive stimuli to elicit weak firing fields. To detect those weak fields, we assumed that a sensitive algorithm would be required. With this in mind, we developed a Bayesian algorithm that takes in spike data and computes a probability distribution for field locations. The algorithm models the data as coming from two sources: (1) a Gaussian that represents the cell's spatially specific firing field; and (2) a uniform non-zero floor that represents the cell's tendency to produce noisy spikes anywhere in the animal's environment (Supplementary Fig. 3a). The algorithm uses an iterative procedure, evaluating each $(x, y)$ coordinate in the environment in $1 \mathrm{~cm}$ bins to determine which locations most likely host the cell's firing field. On simulated data where the ground truth position of the field was known, performance of the algorithm was nearly perfect across a wide range of noise levels for field sizes expected to be found in electrophysiological data (fields with 500 or 250 spikes had a mean error of $0 \mathrm{~cm}$ in a $100 \times 100 \mathrm{~cm}^{2}$ box; fields with 125 spikes had a mean error of $2.35 \mathrm{~cm}$; the number of spikes drawn from noise was varied between $0,500,5000,25,000,50,000$ and 100,000 ) (Supplementary Fig. 3c). On real data, the algorithm successfully identified OV cells with single (Supplementary Fig. 3d, i, ii and iii) and multiple fields (Supplementary Fig. 3d, iv).

\section{A 2-dimensional surface produces strong vectorial responses in OV cells}

Since previously we probed OV responses using discrete, 3D objects ${ }^{23}$ we began by asking whether a flat 2D surface would be sufficient to generate vectorial responses. We used the original Duplo tower object (Supplementary Fig. 2b) and varied its volume by either gradually embedding it into the wall of the recording box or extending it out from the wall. Using a step-like procedure, we changed the object from a fully exposed 3D object, through a partly embedded object ( $50 \%$ standing out from the wall), to a flat 2D surface continuous with the box wall, or vice versa (Fig. 2a). In this experiment, as well as in further parametric experiments, we limited analysis to pre-selected OV cells, identified with a freestanding 3D object, using the criteria defined above. For these cells, we calculated two measures of responsiveness: (1) The firing rate inside a circular region of interest (ROI) in which we expected the cell to fire based on the cell's vector coordinates; and (2) The cell's OV score ${ }^{23}$. For measure (1), the centre of the ROI was the location of the OV field identified with the Bayesian algorithm. This was taken as the maximum of the posterior probability distribution from the 'Object' trial originally used to identify the cell as an OV cell (Fig. 1a, middle). The size of the ROI was always $15 \mathrm{~cm}$, a pre-specified parameter. We confirmed that in all cases the results were independent of this parameter choice (Supplementary Fig. 4). For measure (1) we normalised the data for each cell, by expressing firing rates as a fraction of the maximum value observed for each cell ( $1=$ maximum, $0=$ minimum).

The presence of objects significantly influenced the OV cells' firing rate inside the ROI (Fig. 2c, left) ( $H=12144, p=1.70 \times 10^{-6}, \mathrm{n}=30$ cells, Kruskal-Wallis test). All objects, including the plain 2D surface, produced an increase in firing rate inside the ROI relative to the Empty Box trial (2D vs. Empty Box, $W=50, p=2.92 \times 10^{-4} ; 50 \%$ vs Empty Box, $W=67, p=0.0011 ; 3 \mathrm{D}$ vs. Empty Box, $W=58,5.63 \times 10^{-4}$; two-sided Wilcoxon signed rank tests). The response to the $2 \mathrm{D}$ object was indistinguishable from the response both to the 3D object and the partially embedded object (2D vs. $3 \mathrm{D}, W=252, p=0.4557$; $2 \mathrm{D}$ vs. $50 \%, W=256, p=0.4051$, two-sided Wilcoxon signed rank tests). There was no significant effect of the object on firing rate outside the ROI (Fig. 2c, right) 
( $H=336, p=0.7842, \mathrm{n}=30$ cells, Kruskal-Wallis test). The order of the change - from 3D to 2D, or vice versa - had no effect on the cells' firing rates in the ROI ( $n=14$ experiments from 3D to 2D; $n=27$ experiments from 2D to 3D; $W=319, p=0.1001$, two-sided Wilcoxon signed rank test). The presence of objects also significantly influenced the cells' OV scores (Fig. 2d; Kruskal-Wallis test, $H=0, p=6.54 \times 10^{-8}, \mathrm{n}=30$ cells). All objects $-3 \mathrm{D}$ or $2 \mathrm{D}$ - produced a significant increase in the OV score relative to baseline (2D vs. empty, $W=94, p=0.0044 ; 50 \%$ vs empty, $W=$ $35, p=4.86 \times 10^{-5}$; 3D vs. Empty Box, $, W=30, p=3.11 \times 10^{-5}$; two-sided Wilcoxon signed rank tests). The OV score was larger for both the 3D object and the partially embedded object compared to the $2 \mathrm{D}$ object $\left(2 \mathrm{D}\right.$ vs. $3 \mathrm{D}, W=126, p=3.07 \times 10^{-5}, 2 \mathrm{D}$ vs. $50 \%, W=$ $82, p=0.0020$, two-sided Wilcoxon signed rank tests).

To determine how generally the findings above apply to 2D surfaces, we next tested the cells' response to transparent 2D surfaces (Fig. 3a). In this experiment, the "object" was a transparent film covering a hole in the box wall, with the hole cut to the same size as the Duplo tower $(40 \times 8 \mathrm{~cm})$ (Supplementary Fig. 2c). OV cells responded robustly to this object as well (Fig. 3c, left; Transparent vs. Empty Box, $W=9, P=0.0081$, two-sided Wilcoxon signed rank test), although less strongly than to the 3D Duplo tower (3D vs. Transparent, $W=0, p=2.44 \times 10^{-4}$, two-sided Wilcoxon signed rank test). As before, the effect on firing was not present outside the ROI (Fig. 3c, right) ( $H=0, p=0.4214, \mathrm{n}=$ 14 cells, Kruskal-Wallis test). In addition, the transparent object increased the OV score (Transparent vs. Empty Box, $W=0, p=0.0166$, two-sided Wilcoxon signed rank test) to a level indistinguishable from the 3D object (Fig. 3d) (Transparent vs. 3D, $W=26, p=0.1040$, two-sided Wilcoxon signed rank test). Taken together, the results demonstrate that OV cells respond to a variety of flat $2 \mathrm{D}$ surfaces, often as strongly as they respond to discrete 3D objects.

\section{A visual contrast on the wall is sufficient to elicit vectorial responses in OV cells}

Having shown that OV cells can use 2D surfaces as reference points, we next asked whether the surface could be stripped down further to isolate the underlying features that prompt OV cells to respond. Given that vision likely has an important role in OV firing (see Discussion) we wondered whether a visual contrast - the most primitive kind of 2D object in the present study - could be sufficient to generate vectorial responses. To probe responses to visual contrast, we used a band of self-adhesive tape printed on the wall of the recording chamber (Supplementary Fig. 2d). Again, in a step-like procedure, we changed the contrast from $0 \%$ (no self-adhesive tape), through $10 \%$ and $60 \%$, to $100 \%$ whiteness ('Empty', 'Dark Gray', 'Gray' and 'White', respectively) (Fig. 4a). While some cells had firing rates that clearly increased with visual contrast (Fig. 4b) other cells were more difficult to evaluate by eye (Supplementary Fig. 5a), suggesting that we had approached the lower bound of the cells' preferred stimuli.

There was a significant effect of contrast on the firing rate of OV cells inside the ROI (Fig. 4c, left) ( $H=720, p=0.0177, \mathrm{n}=14$ cells, Kruskal-Wallis test). OV cells showed a significant response to the white contrast ( $W=6, P=0.0034$ ) but only a non-significant trend to a response to the gray contrast $(W=19, P=0.0803)$ and the dark gray contrast $(W=23, P=0.1272)$. No effect of contrast was present outside the ROI (Fig. 4c, right) ( $H=120, p=0.6310, \mathrm{n}=14$ cells, KruskalWallis test). While contrast did not have a statistically significant effect on the OV score (Fig. 4d) ( $H=0, p=0.4112, \mathrm{n}=14$ cells, Kruskal-Wallis test) we note that, for $2 \mathrm{D}$ objects, the OV score suffers from a binning problem since the animal can only explore half of all allocentric angles $\left(180^{\circ}\right.$ out of the full $360^{\circ}$ ). This makes the firing rate inside the ROI a more sensitive measure for these types of features. Taken together, our results establish that a simple visual feature such as contrast modulates the firing rate of OV cells.

\section{The probability that OV cells respond to different features and objects}

The post-hoc statistical tests used above, while advantageous in many respects, have limitations when it comes to comparing responses to different visual contrasts. For example, the response to the white 
contrast was found significant and the response to the gray contrast insignificant, but this makes it difficult to compare: (i) how much more likely is it that OV cells respond to the white contrast, compared to the gray contrast? (ii) how much higher is the likely range of firing rate values for the white contrast, compared to the gray contrast? For this reason, we conducted a complementary statistical analysis that quantifies for every visual contrast (i) the probability that OV cells respond; (ii) a credible region that expresses "there is a $95 \%$ probability that OV cells respond with an average firing rate (FR) between [ $a=$ lower bound] and [ $b=$ upper bound]". Measures (i) and (ii) can be readily calculated with a Bayesian statistical analysis, a method based on classical work ${ }^{24-26}$ and well-advanced in many fields ${ }^{27-29}$. Given the subtle responses in the visual contrast experiment (Fig. 4), the analysis is especially informative here, but we performed the analysis for all experiments of the present study (Fig. 5).

To perform the analysis, we calculated the probability distribution $\mathrm{P}(\mathrm{FR} \mid \mathrm{D})$, the probability that $\mathrm{OV}$ cells respond with a particular average firing rate (FR) to some object (for example, the white contrast) given the data collected (D). By Bayes' theorem, this is given by

$$
P(F R \mid D) \propto P(D \mid F R) P(F R) \quad \text { Eq. } 1
$$

where $P(D \mid F R$ ) is the likelihood and $P(F R)$ is the prior (Fig. 5a). We assigned a Gaussian distribution for the likelihood and a uniform distribution for the prior (see Method for details). To develop some intuition for the method, note that if the data clustered around $2.8 \mathrm{~Hz}$, the dataset would be likely to have been produced by a Gaussian with mean at $3 \mathrm{~Hz}$, but unlikely to have been produced by a Gaussian with mean at $20 \mathrm{~Hz}$. Using the notation above, $P(D \mid F R=3 \mathrm{~Hz})>P(D \mid F R=20 \mathrm{~Hz})$. The main idea behind the method is to systematically move the Gaussian across the real number line (e.g. from firing rate changes of $-10 \mathrm{~Hz}$ to $10 \mathrm{~Hz}$ in steps of $0.01 \mathrm{~Hz}$ ) in order to find which mean values are most consistent with the dataset obtained. This gives a probability distribution for different levels of responding to each object (Fig. 5b-d). More specifically, the data (the firing rate inside each OV cell's ROI) are expressed relative to the 'Empty Box' session,

FR inside ROI in experimental condition - FR inside ROI in 'Empty Box' condition

Eq. 2

which means that in the probability distributions, probability mass on the right (left) of $0 \mathrm{~Hz}$ should be interpreted as a positive (negative) response to the object.

The results from this analysis are summarised in the probability distributions (Fig. 5b-d), showing the probabilities of different average firing rates, for each specific object or feature. For the objects in the first set of experiments, where we varied the discreteness of the object, all curves were in the positive range, occupying locations to the right of $0 \mathrm{~Hz}$ (Fig. 5b). Thus, the probability that $\mathrm{OV}$ cells respond with a positive change in FR was 1 ('3D'), 1 ('50\%') and 1 ('2D'), respectively (Fig. 5b; 95\% probability that response was between [1.12 Hz, 2.62 Hz], [1.22 Hz, 3.06 Hz] and [1.35 Hz, 2.96 Hz]). Consistent with OV cells responding as strongly to $2 \mathrm{D}$ surfaces as $3 \mathrm{D}$ objects, the curves were overlapping (Fig. 5b). Similarly, the probability that OV cells respond to the transparent object was 0.9987 and 1 for the 3D tower in the same experiment (Fig. 5c). However, the strength of the response was lower for the transparent object (with $95 \%$ probability between 0.47 and $2.15 \mathrm{~Hz}$ ) than the normal 3D object (with 95\% probability between 2.81 and $4.29 \mathrm{~Hz}$ ). The separation between the curves makes this difference in responding evident (Fig. 5c).

Visual contrast had an incremental effect on the probability distributions (Fig. $5 \mathrm{~d}$ ). For the dark grey contrast, only a moderate amount of probability $(0.77)$ was above $0 \mathrm{~Hz}$. For the grey contrast, a larger amount of probability (0.89) was above 0 . For the white contrast, nearly all probability mass (0.9997) was in the positive range. That is, the probability that OV cells respond to the white contrast is nearly 1. The trend is seen visually as a right-shift of the curves (Fig. $5 \mathrm{~d}$ ). A similar trend was present when 
applying the same analysis to the OV score (Supplementary Fig. 6a-c). We found that, with 95\% probability, OV cells respond to the white contrast with an average FR between 0.66 and 2,37 Hz. The corresponding ranges for the gray and dark gray contrasts were $[-0.55 \mathrm{~Hz}, 2.51 \mathrm{~Hz}]$ and $[-0.67 \mathrm{~Hz}, 1.53$ $\mathrm{Hz}]$, respectively. Taken together, these results show that OV cells are likely to respond to the grey contrast, but almost certainly respond to the white contrast. This establishes that a visual contrast is sufficient for OV cells to respond, with increasing certainty for higher contrasts. The results were insensitive to the choice of prior (Supplementary Fig. 7) and likelihood function (Supplementary Fig. 8).

\section{Discussion}

In this study, we have identified some of the simplest types of objects and features that make up the extensive stimulus space of OV cells. Using a parametric approach, we show that the cells' capability to encode vectors to discrete objects extends to 2-dimensional, as well as transparent, visual stimuli. In the most extreme case, a simple visual contrast was sufficient to elicit OV firing. The OV response increased gradually as we increased the contrast of the object. Since low contrast features either failed to elicit a response, or only elicited a weak response, while high contrast features consistently succeeded, visual contrast changes are probably among the minimum and fundamental stimulus changes that excite OV cells.

The results show that vision is sufficient to drive responses in OV cells. Combined with our previous finding of impaired object-vector firing in darkness ${ }^{23}$, this shows that vision is both necessary and sufficient to prompt OV responses. This contrasts with border cells $5^{5,9,10}$ in which blockade of the animal's trajectory be necessary since the cells do not respond to elevated borders ${ }^{23}$. Interestingly, boundary-vector (BV) cells in the subiculum respond to gaps in the environment that the animal can traverse ${ }^{14,15}$, raising the possibility that the underlying mechanism generating firing in border cells and $B V$ cells is different ${ }^{23}$. While a fundamental stimulus feature for firing in OV cells is identified, it is still unknown what the equivalent minimal features for border cells and BV cells are.

A further striking difference between these functional cell types is the spectrum of distances they encode. In OV cells ${ }^{23}$ and BV cells ${ }^{13-15}$ firing fields cover both proximal and distal locations, while border cells have only proximal firing fields ${ }^{5,9,10}$. This means that OV cells and BV cells can represent the animal's position relative to discrete objects and features (in the case of OV cells) and relative to boundaries (in the case of BV cells) whether these items are near or far. Border cells represent nearby boundaries that obstruct the animal's immediate path ${ }^{23}$. The distinct natures of these cell types - in terms of (1) distance tuning and (2) object selectivity - provide a complementary and rich representation of the environmental layout, regardless of which specific objects, features and boundaries are present in the environment. Despite their differences, the computational goal of supporting landmark-based navigation is likely shared between OV cells, BV cells and border cells ${ }^{15}$.

The finding that a visual contrast alone is sufficient for OV cells to respond suggests that even when no salient object is present in the environment (such as in the 'Empty Box' trial) OV cells might respond to corners, edges, shadows and other types of contrast. Consistent with this, OV cells are rarely silent in the 'Empty Box' trial but often show one or more firing fields (Supplementary Fig. 5b and c) - usually weaker than the object-induced field. Without other knowledge it would be easy to disregard this firing as neuronal 'noise' ${ }^{30}$. However, the present results suggest that this firing might not be 'noise' but a real effort by OV cells at signaling the presence of the few landmarks available in a nearly content-free environment. 
What implications does this have for spatial representation in MEC in general? Given the substantial number of OV cells ${ }^{23}$ and given the present results, researchers that record from MEC in the absence of discrete free-standing objects, could expect to find spatial firing fields simply because of the sensitivity of OV cells to visual contrasts. These firing fields would be (1) spatial, if the cues remain in the same place; and (2) non-periodic, because the cues are not periodic. From this perspective, our findings may explain the presence of 'aperiodic spatial cells' in MEC recorded by us and others ${ }^{31-35}$ : these are cells with significant spatial or positional information that are not grid cells. In one study, the fraction of such MEC cells was estimated at $68 \%{ }^{33}$. The present results raise the possibility that a fraction of these aperiodic spatial cells are OV cells respondingto visual contrasts such as corners or shades within an otherwise stimulus-deprived environment.

We have previously reported that aperiodic spatial cells depend on activity of somatostatin (SOM) interneurons while grid cells depend on parvalbumin (PV) interneurons ${ }^{35}$. Now, if the proposal that aperiodic spatial cells are OV cells is correct, this would imply (1) that OV cells require activity of SOM interneurons and (2) that OV cells and grid cells are modulated by distinct subclasses of inhibitory interneurons. While this possibility has not yet been tested, distinct inhibitory control of OV cells and grid cells would further strengthen the notion that spatial representation in MEC is of a dual nature. That is, spatial representation in MEC might be functionally separated into two distinct systems: one involving grid cells, speed cells and HD cells (dependent on PV interneurons via grid cells and speed cell $\mathrm{s}^{35}$ ) for self-motion-dependent representation in open spaces ${ }^{2,36}$ and one involving OV cells and border cells (putatively dependent on SOM interneurons via OV cells) for more landmark-based navigation. 


\section{Methods}

Subjects. Data were obtained from 7 wild-type C57/BL6 mice (6 males, 1 female) aged 3-12 months. All mice were kept on a $12 \mathrm{~h}$ light/12 h dark schedule in a humidity and temperature-controlled environment. The mice were housed in single mouse cages after implantation. Experiments were performed in the dark phase. The mice were not deprived of food or water. Experiments were performed in accordance with the Norwegian Animal Welfare Act and the European Convention for the Protection of Vertebrate Animals used for Experimental and Other Scientific Purposes.

Surgery and electrode implantation. The mice were anaesthetized with $5 \%$ isoflurane (air flow: 1.2 $\mathrm{I} / \mathrm{min}$ ) in an induction chamber. After induction of anaesthesia, they received subcutaneous injections of buprenorphine (Temgesic) and meloxicam (Metacam). The mice were then fixed in a Kopf stereotaxic frame for implantation. The local anaesthetic bupivacaine (Marcaine) was injected subcutaneously before the incision was made. During surgery, isoflurane was gradually reduced from $3 \%$ to $1 \%$ according to the animal's physiological condition. The depth of anaesthesia was monitored by testing tail and pinch reflexes as well as breathing. Anaesthetized mice were implanted with a single bundle of four tetrodes attached to a microdrive fastened to the skull of the mouse. The tetrodes were targeted to MEC at an angle of $3^{\circ}$ relative to the bregma/lambda horizontal reference plane, with tips pointing in the posterior direction. The tetrodes were inserted 3.2-3.3 mm lateral to the midline and $0.4 \mathrm{~mm}$ anterior to the transverse sinus edge, with an initial depth of $900 \mu \mathrm{m}$. The implant was secured to the skull with histoacryl and dental cement. One screw was connected to the drive ground. Tetrodes were constructed from four twisted $17-\mu \mathrm{m}$ polyimide-coated platinum-iridium (90-10\%) wires (California Fine Wire). The electrode tips were plated with platinum to reduce electrode impedances to between 120 and $220 \mathrm{k} \Omega$ at $1 \mathrm{kHz}$.

Recording procedure. Data collection started 1-2 weeks after implantation of tetrodes. During recording, the animal was connected to an Axona data acquisition system (Axona) via an AC-coupled unity-gain operational amplifier close to the animal's head, using a lightweight counterbalanced multiwire cable from both implants to an amplifier. Unit activity was amplified 3,000-14,000 times and band-pass filtered between 0.8 and $6.7 \mathrm{kHz}$. Triggered spikes were stored to disk at $48 \mathrm{kHz}$ with a 32bit time stamp. An overhead camera recorded the position of two light-emitting diodes (LEDs) on the head stage, each at a sampling rate of $50 \mathrm{~Hz}$. The diodes were separated by $3 \mathrm{~cm}$. To sample activity at multiple dorsoventral locations, the tetrodes were lowered in steps of $25-50 \mu \mathrm{m}$. Recordings started when the tetrodes were judged to be in the MEC, using theta modulation, presence of spatial and directional cell types and as well as recording depth as criteria.

Spike sorting and cell classification. Spike sorting was performed offline using graphical cluster-cutting software (ctools, T. Waaga). Spikes were clustered manually in 2D projections of the multidimensional parameter space (consisting of waveform amplitudes), using autocorrelation and cross-correlation functions as additional separation tools and separation criteria. Cluster separation was assessed by calculating distances between spikes of different cells in Mahalonobis space ${ }^{37}$ (median isolation distance: $16.3 ; 25$ th and 75 th percentiles: $11.1-26.8$ ). Noise in the vicinity of clusters was expressed as the $\mathrm{L}$ ratio ${ }^{37}$ (median $\mathrm{L}$ ratio: $0.18 ; 25$ th and 75 th percentiles: $0.05-0.78$ ). Clusters on successive recording sessions were identified as the same unit if the locations of the spike clusters were stable.

Behavioural procedures. The mice were trained to forage for cookie crumbs in an $80 \mathrm{~cm} \times 80 \mathrm{~cm}$ square arena, enclosed by $50-\mathrm{cm}$-high walls. Cookie crumbs were thrown out one-by-one in random locations. Thick dark blue curtains surrounded the recording arena, except for an opening on one side. Before testing, the mouse rested outside the curtain in a plexiglass cage coated with towels. Testing 
was performed at low light levels to encourage exploration. Between trials, the mat covering the floor of the recording box was cleaned.

Screening for object-vector cells. To screen for object-vector (OV) cells we first performed an 'Empty Box' trial in which no object was present in the arena, followed by an 'Object' trial in which a towershaped Duplo object (Supplementary Fig. 2a) was placed in the center of the arena. In OV cells, the 'Object' trial was expected to induce new firing compared to the 'Empty Box' trial. To verify that firing was in a specific distance and direction away from the object, we performed a third 'Moved Object' trial in which the object was moved in a pseudo-random fashion to a new location. Trials were typically spaced by a few minutes, during which the experimenter clustered and inspected recorded cells. The identity of the object used for screening trials was the same from day to day. Most trials were 30 minutes long (mean recording time 29.3 minutes).

$2 D / 3 D$ experiment. To determine whether OV cells produce vectorial responses to flat $2 \mathrm{D}$ surfaces, and whether they are comparable to responses to the discrete 3D objects used in previous work ${ }^{23}$, we used a step-like experimental protocol in which the discreteness of an object was varied (Fig. 2). In the first trial, a tower-like Duplo object $(40 \times 8 \times 8 \mathrm{~cm})$ was completely embedded into the wall of the recording arena through an opening with the same height and length as the object $(40 \times 8 \mathrm{~cm})($ Supplementary Fig. 2b). Thus, in this trial, the object was a flat 2D surface continuous with the wall (' $2 \mathrm{D}$ '). In the second trial, the same object was only partially embedded so that half of its volume was exposed to the animal ('50\%'). In the third trial, the entire volume of the object was exposed to the animal ('3D'). The width of object extending into the arena was $0,4 \mathrm{~cm}$ and $8 \mathrm{~cm}$, respectively. Most experiments $(n=27)$ were performed in the direction from $2 \mathrm{D}$ to $3 \mathrm{D}$ as above, but we also performed experiments $(n=14)$ in the opposite direction, from 3D to 2D. Most trials were 30 minutes long (mean recording time 30 minutes).

Transparent-object experiment. To verify the findings from the 2D/3D experiment and confirm that a variety of flat 2D surfaces can induce vectorial responses in OV cells, we performed an additional experiment with transparent 2D objects (Fig. 3). The experiment began with a reference 'Empty Box' trial without an object present. In the second trial, an opening in the arena wall (height and length $40 \times 8 \mathrm{~cm}^{2}$ ) was covered with transparent film to produce an opaque 2D surface (Supplementary Fig. $2 \mathrm{c})$. The behaviour of the mice suggested that they noticed the presence of this "object". During the trial, dark blue curtains surrounded the arena (curtains located about $1 \mathrm{~m}$ away from the arena walls), preventing the animal from seeing any other material or object than the uniform curtain behind the transparent surface. In the third trial, a tower-like Duplo object was placed in the arena, in the same location where the transparent object had been. The purpose of the last trial was to compare any firing to the transparent object to firing to a standard object known to elicit strong vectorial responses. Most trials were 30 minutes long (mean recording time 30 minutes).

Contrast experiment. Having shown that 2D surfaces are sufficient to elicit vectorial responses, we asked whether the fundamental stimuli that elicit activity in OV cells are visual contrasts (Fig. 4). The experiment began with an 'Empty Box' trial with no visual contrast present. In the next three trials ('Dark Gray', 'Gray' and 'White', respectively) the degree of whiteness of a visual contrast on the arena wall was varied between $0 \%, 10 \%, 60 \%$ and $100 \%$. The visual contrast was a $40 \times 8 \mathrm{~cm}^{2}$ band of selfadhesive tape printed on the arena wall (Supplementary Fig. $2 \mathrm{~d}$ ). The printed material had the same texture as the rest of the arena wall. The arena wall on which the contrast was put (N, S, E, or W wall) varied depending on the OV cell's properties. For example, if the OV cell had a firing field north of the object, the visual contrast would be placed on the south wall. Changes in location for the contrast cue were made by rearranging the locations of the entire walls. Most trials were 30 minutes (mean recording time 28.2 minutes). 
Firing rate maps. To produce firing rate maps (Fig. 1a), position estimates were convolved with a 35point Gaussian filter and $x, y$-coordinates were sorted into $2 \mathrm{~cm} \times 2 \mathrm{~cm}$ bins. Spike timestamps were matched with position timestamps. Firing rate maps were determined by counting the number of spikes falling in each bin and dividing by the amount of time spent in that bin. The maps were subsequently smoothed with a 2D Gaussian kernel with s.d. of 2 bins $(4 \mathrm{~cm})$ in both the $x$ and the $y$ directions.

Spatial information content. To calculate the spatial information content (Fig. 1c, e) we used the cell's firing rate map to compute the spatial information rate ${ }^{38}$ as

$$
\sum_{i=1}^{N} p_{i} \frac{\lambda_{i}}{\lambda} \log _{2}\left(\frac{\lambda_{i}}{\lambda}\right)
$$

where $\lambda_{i}$ is the mean firing rate in the ith bin, $\lambda$ is the overall mean firing rate and $p_{i}$ is the probability of the mouse being in the ith bin (time spent in the ith bin/total recording time). Spatial information content in bits per spike was obtained by dividing the information rate by the mean firing rate of the cell.

Object-vector score. To calculate the object-vector (OV) score (Fig. 1d, f) we first calculated firing rate maps that expressed the cell's firing as a function of the animal's distance $(\mathrm{cm})$ and orientation (degrees) from the object (Fig. 1b) ('vector-maps' in ref 23). For this, we sorted spikes and position estimates into distance bins of $2 \mathrm{~cm}$ and orientation bins of 10 degrees. The firing rate in each bin was calculated by dividing the number of spikes by the amount of time spent in the bin. The maps were smoothed with a 2D Gaussian kernel with s.d. of 1.5 bins ( $3 \mathrm{~cm}, 15$ degrees). Because orientation is a circular variable, we stacked three maps vertically before smoothing to ensure smoothing of edges at 0 and 360 degrees. In these maps, East (North) relative to the room's frame was defined as 0 degrees (90 degrees). Having computed these firing rate maps, the OV score was defined as the Pearson correlation between maps from the 'Object' and 'Moved Object' trials (Fig. 1b). When the cell's firing depends on the animal's position relative to the object, rather than the animal's absolute position, the correlation should be high.

Shuffling of spike data. In order to identify OV cells with (1) spatial firing patterns and (2) vectorial firing patterns locked to the object, we required thresholds (i.e. cutoff values) for (1) spatial information content and (2) the OV score. To find such thresholds, we implemented a standard shuffling procedure. To shuffle the spikes of the cell, the entire sequence of spike timestamps was shifted in time relative to the mouse's path by a random number. The random number was drawn from a uniform distribution such that

$$
p\left(t_{\text {shift }}\right)=\frac{1}{b-a}
$$

where

$$
b=\text { session length }-20 s
$$$$
a=20 s
$$

For all $t_{\text {shift }}$ such that 
The shifted spike timestamps were wrapped around from the end of the trial, ensuring that all shuffled spikes occurred at some point during the trial. Time shifts varied randomly between permutations and cells.

We performed cell-by-cell shuffling 33,39 rather than global shuffling $23,40,41$. For each cell, we performed 200 shuffles of the cell's spike timestamps from the 'Object' trial. We then calculated the spatial information content for each one of these shuffles, yielding the cell-specific shuffling distribution (Fig. 1c). The 99th percentile of this shuffling distribution was taken as the cell-specific threshold. That is, cells with actual spatial information content $>$ the 99th percentile of the shuffling distribution were considered spatially modulated cells (Fig. 1e). Almost the same procedure was implemented for the OV score, with the only exception that we shuffled spike timestamps from both the 'Object' and 'Moved Object' trials separately, generating pairs of shuffled cells. We then calculated the OV score based on each pair of shuffles.

Definition of object-vector cells. As in earlier work ${ }^{23}$, cells had to satisfy multiple criteria to classify as OV cells:

(0) (Pre-selection criterion for new fields) Since objects should induce the presence of new firing fields in OV cells, we required one or more new fields to appear in the 'Object' trial compared to the 'Empty Box' trial. For this, we used the Bayesian field detection algorithm to identify all fields present in the 'Empty Box' and 'Object' trials. We then compared distances between field centers in the two trials. Fields with centers less than $2 \mathrm{~cm}$ apart were considered the same field. Using this procedure, we verified that each cell had at least one new field in the 'Object' trial.

(1) (Spatial information criterion) Since OV cells should show spatial tuning when objects are present in the environment, we required the spatial information content in the 'Object' trial to exceed a cell-specific threshold. This cell-specific threshold was the 99th percentile of the cell's own shuffling distribution (Fig. 1c). We created this shuffling distribution by shifting the cell's spike timestamps 200 times (see "Shuffling of spike data") and calculating the spatial information for each one of the shuffled cells.

(2) (OV score criterion) Since the OV score quantifies how strongly the cell's firing depends on the animal's distance and orientation from the object (see "Object-vector score") we required the OV score to exceed a cell-specific threshold. The threshold was the 99th percentile of the cell's own shuffling distribution (Fig. 1d). We created this distribution by separately shifting the cell's spike timestamps 200 times in the 'Object' and 'Moved Object' trials and calculating the OV score for each shuffled pair.

(3) (Field distance criterion) In order for the cells to be 'vectorial', the cell's firing must be offset from the object rather than located directly at the object. Therefore, as in previous work ${ }^{23}$, we required that $\mathrm{OV}$ cells had fields offset from the object's center by more than $4 \mathrm{~cm}$. To implement this criterion, we detected firing fields on the 'Object' trial and measured the distance of their centers from the object. Only cells that met the $4 \mathrm{~cm}$ distance criterion were kept. We note that cells that fire directly at the object are rare in MEC. In this dataset, we observed only a single cell that passed all other criteria for OV cells but failed to pass the distance criterion.

Field detection algorithm. Since the aim of the study was to investigate the most basic features of objects that produce activity in OV cells, we expected some primitive stimuli to elicit weak firing fields. Consistent with this, in many OV cells, we found that stimuli consisting of visual contrasts produced firing fields that were barely noticeable by eye (Supplementary Fig. 5a). To detect weak firing fields, in the presence of background noise, we required a sensitive field detection algorithm. For this purpose, 
we developed a Bayesian field detection algorithm (Supplementary Fig. 3). Note that the OV score is independent of this algorithm, but OV classification depended on the algorithm via steps (0) and (3) above (see "Definition of object-vector cells").

\section{Step 1. Probability distribution for field locations.}

The overall goal can be described by Bayes' theorem. We want to calculate

$$
P\left(\text { field }_{x, y} \mid D\right)=\frac{P\left(D \mid \text { field }_{x, y}\right) P\left(\text { field }_{x, y}\right)}{P(D)}
$$

where we define

$$
\text { field }_{x, y} \equiv \text { the cell's firing field lies at the location } \mathrm{x}, \mathrm{y}
$$

$$
D \equiv \text { the cell's spike data }
$$

In words, we want to calculate the probability that the cell's field lies at a particular location $(x, y)$ given the spike data of the cell. This is given by the likelihood that the cell's spikes were produced by a field lying at this location (the term $P\left(D \mid\right.$ field $\left._{x, y}\right)$, multiplied by the prior probability that the field lies at this location (the term $P\left(\right.$ field $\left._{x, y}\right)$ ), divided by a normalizing constant (the term $P(D)$ ).

Our goal in step 1 is to calculate a probability distribution for the field locations, which requires us to assign a likelihood and choose a prior. For the likelihood, which is a model for the process that generates the data, we assume that spikes originate from two sources: (a) a 2-dimensional Gaussian that represents the cell's firing field and (b) a uniform non-zero floor that represents the cell's tendency to produce noisy spikes anywhere in the environment (Supplementary Fig. 3a).

All positions in the animal's environment can be represented in discrete steps (we used $1 \mathrm{~cm}$ bins) by

$$
\text { pos }=\left(\begin{array}{c}
\operatorname{pos}_{1} \\
\operatorname{pos}_{2} \\
\vdots \\
\operatorname{pos}_{N}
\end{array}\right)=\left(\begin{array}{cc}
x_{1} & y_{1} \\
x_{2} & y_{2} \\
\vdots & \vdots \\
x_{N} & y_{N}
\end{array}\right)
$$

We want the algorithm to calculate the probability that the spike data originate from a firing field at each of the locations $\operatorname{pos}_{1}, \operatorname{pos}_{2} \ldots \operatorname{pos}_{\mathrm{N}}$. Each iteration of the algorithm corresponds to calculating the likelihood for one such location.

For $\operatorname{pos}_{1}=\left(x_{1}, y_{1}\right)$ (first row of the matrices above), the first iteration of the algorithm proceeds as follows. Let the spike data be represented by

$$
D=\left(\begin{array}{c}
d_{1} \\
d_{2} \\
\vdots \\
d_{n}
\end{array}\right)=\left(\begin{array}{cc}
x_{1}^{\text {spikes }} & y_{1}^{\text {spikes }} \\
x_{2}^{\text {spikes }} & y_{2}^{\text {spikes }} \\
\vdots & \vdots \\
x_{n}^{\text {spikes }} & y_{n}^{\text {spikes }}
\end{array}\right)
$$

where the first column represents the x-coordinate at which each spike occurred and the second column represents the $y$-coordinate. First, we will apply a selector function that picks out a subset of all spikes, i.e., the function will select spikes that occurred in a circle (of radius $r$ ) centered around pos $s_{1}$ $=\left(x_{1}, y_{1}\right)$. This step ensures that the results are not influenced by spikes that occurred far away from the current location $\operatorname{pos}_{1}=\left(\mathrm{x}_{1}, \mathrm{y}_{1}\right)$. More precisely, we use the reduced spike matrix 


$$
\widehat{D}=\left(\begin{array}{c}
\widehat{d_{s}} \\
\vdots \\
\widehat{d_{s+m}}
\end{array}\right)
$$

455

456

where

$$
\hat{d}_{i}=d_{i}
$$

For all $d_{i}$ such that

$$
\sqrt{\left(d_{i}-\operatorname{pos}_{1}\right)\left(d_{i}-\operatorname{pos}_{1}\right) \mathrm{T}}<r
$$

where we chose $r=15 \mathrm{~cm}$. That is, $\widehat{d_{s}}$ is the first spike that falls within a circle of radius $r$ centered at $\operatorname{pos}_{1}$ and $\widehat{d_{s+m}}$ is the last spike that falls within the circle. $m$ is the total number of spikes that fall within the circle.

Using only the spikes in a circle around pos ${ }_{1}$, we calculate the likelihood as

$$
\begin{aligned}
\mathcal{L}\left(\operatorname{pos}_{1}\right)=(b & \left.+\exp \left(-\left(\widehat{d_{s}}-\operatorname{pos}_{1}\right) \frac{\Sigma^{-1}}{2}\left(\widehat{d_{s}}-\operatorname{pos}_{1}\right) \mathrm{T}\right)\right) \\
& \times\left(b+\exp \left(-\left(\widehat{d_{s+1}}-\operatorname{pos}_{1}\right) \frac{\Sigma^{-1}}{2}\left(\widehat{d_{s+1}}-\operatorname{pos}_{1}\right) \mathrm{T}\right)\right) \ldots \\
& \times\left(b+\exp \left(-\left(\widehat{d_{m}}-\operatorname{pos}_{1}\right) \frac{\Sigma^{-1}}{2}\left(\widehat{d_{m}}-\operatorname{pos}_{1}\right) \mathrm{T}\right)\right)
\end{aligned}
$$

where $b=1.1$ is a constant that implements the uniform non-zero floor (Supplementary Fig. 3a), $\mathrm{T}$ denotes the transpose and $\Sigma^{-1}$ is the inverse of the covariance matrix, which was the identity matrix. We have assumed independence between data points, allowing us to multiply the terms corresponding to the different data points.

In practice, we calculate the log likelihood

$$
\begin{aligned}
\log \left(\mathcal{L}\left(\operatorname{pos}_{1}\right)\right) & =\log \left(b+\exp \left(-\left(\widehat{d_{s}}-\operatorname{pos}_{1}\right) \frac{\Sigma^{-1}}{2}\left(\widehat{d_{s}}-\operatorname{pos}_{1}\right) \mathrm{T}\right)\right) \\
& +\log \left(b+\exp \left(-\left(\widehat{d_{s+1}}-\operatorname{pos}_{1}\right) \frac{\Sigma^{-1}}{2}\left(\widehat{d_{s+1}}-\operatorname{pos}_{1}\right) \mathrm{T}\right)\right) \ldots \\
& +\log \left(b+\exp \left(-\left(\widehat{d_{m}}-\operatorname{pos}_{1}\right) \frac{\Sigma^{-1}}{2}\left(\widehat{d_{m}}-\operatorname{pos}_{1}\right) \mathrm{T}\right)\right)
\end{aligned}
$$

To evaluate the log likelihood, we subtract the maximum value of the log likelihood as follows

$$
\exp \left(\log \left(\mathcal{L}\left(\operatorname{pos}_{1}\right)\right)-\max \left(\log \left(\mathcal{L}\left(\operatorname{pos}_{1}\right)\right)\right)\right)
$$

which yields the likelihood $\mathcal{L}\left(\right.$ pos $\left._{1}\right)$ that the field is at the location pos ${ }_{1}=\left(\mathrm{x}_{1}, \mathrm{y}_{1}\right)$. Repeating this process for locations $\operatorname{pos}_{2} \ldots \operatorname{pos}_{N}$ gives the full likelihood distribution $\mathcal{L}\left(\operatorname{pos}_{1}\right) \ldots \mathcal{L}\left(\operatorname{pos}_{N}\right)$. As prior, we chose a Gaussian centered at the object location. That is, for $\operatorname{pos}_{1}=\left(\mathrm{x}_{1}, \mathrm{y}_{1}\right)$, the prior probability was

$$
P\left(\text { field }_{x_{1}, y_{1}}\right)=\exp \left(-\left(\text { pos }_{1}-\text { object }\right) \frac{\Lambda^{-1}}{2}\left(\text { pos }_{1}-\text { object }\right) \top\right)
$$


480

481

482

483

484

485

486

487

488

489

490

491

492

493

494

495

496

497

498

499

500

501

502

503

504

505

506

507

508

509

510

511

512

513

514

515

516

517

where object $=\left(x_{\text {object, }}\right.$ Yobject $)$ are the coordinates of the object and $\Lambda=400 \times\left(\begin{array}{ll}1 & 0 \\ 0 & 1\end{array}\right)$. These values were chosen to match the distribution of OV fields observed in previous work ${ }^{23}$. Specifically, the prior accounts for the experimental finding that OV fields are more common near than far away from the object. This property has also been observed for boundary-vector cells ${ }^{14,15,42}$. Finally, we multiply the likelihood by the prior and normalize to find the posterior probability distribution.

\section{Step 2. Extracting field positions from probability distribution}

Once we had acquired a probability distribution for the cell's fields, the aim was to extract field positions from the probability distribution. This step can be implemented in multiple ways, but we used a simple approach based on finding local maxima in the probability distribution. As a preliminary note, suppose that OV cells were known only to have a single firing field. In that case, we would have simply found the maximum of the probability distribution and used the associated $x, y$ coordinates as the field location. That is,

$$
f \widehat{e l d}_{x, y}=\underset{\text { field }_{x, y}}{\operatorname{argmax}}\left(P\left(\text { field }_{x, y} \mid D\right)\right)
$$

where $f \widehat{\text { leld }} x, y$ is the estimated field location. However, we included the possibility that OV cells had two or more firing fields, as known from previous work ${ }^{23}$. For this, we extracted the 50 probability values that maximized the posterior and sorted them in descending order, as follows

$$
p_{1} \geq p_{2} \geq \cdots \geq p_{50}
$$

where $p_{1}$ is the largest value in the probability distribution, $p_{2}$ is the second largest value and so on. We had 50 position coordinates associated with these probabilities, which we denote by $c$

$$
c_{1}, c_{2}, \cdots c_{50}
$$

Some of these coordinates will be neighbors and likely represent the same field. To remove close neighbors, we calculated a Euclidian distance matrix $\mathcal{M}$ where each element $\mathcal{M}_{i j}$ is the Euclidian distance function (denoted by $\mathrm{d}$ ) between coordinates $c_{i}$ and $c_{j}$

$$
\mathcal{M}_{i j}=d\left(c_{i}, c_{j}\right)=\sqrt{\left(c_{i}-c_{j}\right)\left(c_{i}-c_{j}\right) \mathrm{T}}
$$

Because the Euclidian distance function is symmetric, we considered only the upper triangular part of this matrix. We considered two coordinates $c_{i}$ and $c_{j}$ as neighbors if

$$
d\left(c_{i}, c_{j}\right)<10 \mathrm{~cm}
$$

For each probability value, we therefore had a set of neighbors. If a probability value was larger than all its neighbors, that probability value (and the associated $x, y$ coordinates) was taken as an estimated field position. This process was repeated for all probability values until all field positions had been extracted.

\section{Simulations}

In order to verify the algorithm on data where we had ground truth (knew the real field location), we simulated cells with spatial firing fields and noisy background activity (Supplementary Fig. 3b and c). To simulate the firing field, spikes were drawn randomly from a 2D Gaussian centered at some random location in a $100 \times 100 \mathrm{~cm}^{2}$ environment. The number of spikes from the field was $500,250,125,50$ or 15 , depending on the condition. To simulate background noise, we drew spikes from a uniform distribution across the $100 \times 100 \mathrm{~cm}^{2}$ environment. The number of spikes drawn from the noise was 0 , 
$2500,25000,50000$ or 100000 , depending on the condition. Visual inspection confirmed that the algorithm successfully found firing fields even in cases where they were completely obscured by noise (Supplementary Fig 3b, i-iii). To systematically test algorithm performance, we performed 50 repetitions for each condition. To quantify the error of the algorithm, we calculated the Euclidian distance between the algorithm's estimate of the field location and the true field location

$$
\text { error }=\sqrt{\left(\text { estimate }_{x, y}-\text { true }_{x, y}\right)\left(\text { estimate }_{x, y}-\text { true }_{x, y}\right) \mathrm{T}}
$$

The algorithm's estimate was taken as the maximum of the posterior probability distribution

$$
\text { estimate }_{x, y}=\underset{\text { field }_{x, y}}{\operatorname{argmax}}\left(P\left(\text { field }_{x, y} \mid D\right)\right)
$$

Performance was perfect (mean error $0 \mathrm{~cm}$ ) across all levels of noise when the field contained 500 or 250 spikes (Supplementary Fig. 3c). When the field contained 125 spikes, the mean error was lower than $2 \mathrm{~cm}$ for all levels of noise except the largest one (mean error $11.8 \mathrm{~cm}$ ). Large errors were only found for conditions in which the firing field consisted of a small number of spikes (50 or 15 spikes). The chance level $(40.21 \mathrm{~cm}$ ) was taken as the expected error of an algorithm making random (uniformly distributed) estimates of the field location.

\section{Measures of responsiveness of OV cells}

\section{Firing rate inside a circular region of interest (ROI)}

In order to assess how strongly OV cells produce vectorial responses to different stimuli, we used two measures of responsiveness: (1) the firing rate of the cell inside a circular region of interest (ROI) in which we expected the cell to fire based on its vector coordinates; (2) the OV score. For measure (1), we first applied the Bayesian field detection algorithm (Supplementary Fig. 3) to the 'Object' trial to identify the coordinates of the cell's firing fields. By subtracting the object location, we obtained object-referenced coordinates of the firing fields, which we term 'vector coordinates'. The vector coordinates were transferred to the experimental trial (one of the trials in the 2D/3D, transparent or contrast experiment). The ROI was taken as a circle of radius $15 \mathrm{~cm}$, centered at the vector coordinates. For how this parameter choice (size of the ROI) affects the experimental findings, see Supplementary Figure 4. All position samples and spikes that fell within the ROI were extracted. The number of spikes inside the ROI was divided by the amount of time the animal spent inside the ROI, to obtain the firing rate.

\section{The OV score}

For measure (2), we had to calculate firing rate maps that expressed the cell's firing as a function of the animal's distance and orientation to the object (Fig. 1b) (object-centered firing rate maps: see "OV score" for details). The maps were calculated in the 'Object' trial and the experimental trial (one of the trials in the 2D/3D, transparent-object or contrast experiment). We then calculated the OV score for this pair of trials, as the Pearson correlation between the object-centered maps. If the cell's firing in the experimental trial correlated with the firing in the 'Object' trial - that is, if the distance and direction tuning was preserved - the OV score was expected to be high. We note that for 2-dimensional stimuli on the wall of the arena the OV score suffers from a binning problem: the animal can only explore half of all orientation bins around the object $\left(180^{\circ}\right.$ out of the full $\left.360^{\circ}\right)$. For this reason, we give stronger weight to measure (1) than measure (2) in the present study. 


\section{Inferring the probability that OV cells respond to different objects}

In order to compute (1) the probability that OV cells respond to different objects and (2) a range of reasonable firing rate values for each object, we performed a Bayesian statistical analysis (Fig. 5). The method is standard ${ }^{26,27}$ and based on similar principles as the field detection algorithm (Supplementary Fig. 3). Intuitively, if we have data that cluster around $2.8 \mathrm{~Hz}$, the dataset would be likely to have been produced by a Gaussian at $3 \mathrm{~Hz}$, but unlikely to have been produced by a Gaussian at $20 \mathrm{~Hz}$. The idea behind the method is to systematically move the Gaussian across a range of plausible mean values, to see which values are most consistent with the actual data obtained. This assumes a Gaussian as the underlying model for the process of data generation, but we verified that our results were insensitive to this assumption (see below). For ease of illustration, suppose we perform the analysis for the white contrast condition. To compute (1) and (2), we would like to calculate $P(F R \mid D)$ - the probability that OV cells respond with a particular change in firing rate (FR) to the white contrast, given the data (D) obtained (Fig. 2c, 3c, 4c). According to Bayes' theorem, we can write this as

$$
P(F R \mid D)=\frac{P(D \mid F R) P(F R)}{P(D)}
$$

where $P(D \mid F R)$ is the likelihood of a particular firing rate and $P(F R)$ is the prior probability of that firing rate. More precisely, we defined the data $(D)$ as all the firing rate values observed for OV cells inside their ROI (relative to the firing rate in the same ROI in the 'Empty Box' trial)

$$
D=\left(\begin{array}{c}
d_{1} \\
d_{2} \\
\vdots \\
d_{n}
\end{array}\right)
$$

That is, $d_{1}$ is the firing rate of the first OV cell inside its ROI in the 'White contrast' trial (subtracted by the same value in the 'Empty Box' trial), $d_{2}$ is the firing rate of the second OV cell inside its ROI in the 'White contrast' trial (subtracted by the same value in the 'Empty Box' trial). $n$ is the total number of OV cells. For the results presented in Figure 5, we assigned a Gaussian likelihood. The likelihood was calculated as

$$
\left.p(D \mid F R)=\frac{1}{(\sqrt{2 \pi} \sigma)^{n}} \exp \left(-\frac{1}{2} \sum_{i}^{n} \frac{\left(d_{i}-F R\right)^{2}}{\sigma^{2}}\right)\right)
$$

where $F R$ is the mean of the Gaussian and $\sigma$ is the standard deviation. $F R$ is the parameter we try to infer - the estimated change in the firing rate for the white contrast (compared with the 'Empty Box' session). The key question of interest is, "what are the most probable values of this parameter, given the data?". To choose a value for $\sigma$, we used the standard deviation observed in the real data. Given that most of our OV cells were recorded on different days and in different animals, we assumed independence between data points. This allows for summation over different $d_{i}$ inside the exponential. The calculation above was repeated for values of FR from -10 to $10 \mathrm{~Hz}$ with a step size of $0.01 \mathrm{~Hz}$. That is, we repeated the calculation for all reasonable values of FR. Note that we did not observe firing rate increases of more than $10 \mathrm{~Hz}$ in the real data. We assumed a uniform prior, given by

$$
p(F R)=\frac{1}{b-a}
$$

where $b=10$ was the upper bound of $F R$ and $a=-10$ was the lower bound. That is, all FR values had the same prior probability before seeing the data. Multiplication of the likelihood by the prior, followed by normalization, gave the probability distribution $P(F R \mid D)$. In order to calculate (1) the probability 
that OV cells responded to the object, we calculated the sum of all probabilities to the right of the origin

$$
p(\text { response })=\sum_{F R>0} P(F R \mid D)
$$

In order to calculate (2) a range of reasonable firing rate values for each object, we sorted all probabilities in descending order and summed them until the amount of probability exceeded 0.95. Looking at the FR values associated with these probabilities, we used the minimum FR and the maximum FR as the lower and upper bound, respectively. This gives a $95 \%$ probability range for the true FR ('credible region' ${ }^{27}$ ).

In Supplementary Figure 7, we tested the sensitivity of the results of this analysis to different priors. First, we used a Gaussian prior (Supplementary Fig. 7a). The prior probability was calculated as

$$
p(F R)=\frac{1}{\sqrt{2 \pi} \sigma} \exp \left(-\frac{(F R-\mu)^{2}}{2 \sigma^{2}}\right)
$$

where we set $\mu=0$ an $\sigma=2$.

We then used priors based on the beta distribution (Supplementary Fig. $7 \mathrm{~b}$ and $\mathrm{c}$ ). Because the beta distribution is defined for $x$ on the interval $[0,1]$ we first mapped each value of FR to this interval. That is, the smallest value $F R=-10 \mathrm{~Hz}$ was mapped to 0 , the largest value $F R=10$ was mapped to 1 , and so on. The prior probability was calculated as

$$
p(F R)=p(x)=\frac{\Gamma(\alpha) \Gamma(\beta) x^{\alpha-1}(1-x)^{\beta-1}}{\Gamma(\alpha+\beta)}
$$

where $\Gamma$ is the gamma function. In panel $b$, we used $\alpha=3$ and $\beta=1.5$. In panel $c$, we used $\alpha=1.5$ and $\beta=1.5$.

In Supplementary Fig. 8, we tested the sensitivity of the results of the analysis to different likelihoods. We first used a binomial likelihood (Supplementary Fig. 8a). To compute the binomial likelihood, we first binarized the data by applying the sign function

$$
\operatorname{sign}\left(d_{i}\right)=\left\{\begin{array}{c}
1 \text { if } d_{i}>0 \\
-1 \text { if } d_{i}<0
\end{array}\right.
$$

This gave $r$ successes (number of OV cells with increased firing rate) and ( $n-r)$ failures (number of OV cells with decreased firing rate) in $n$ trials (total number of OV cells recorded). The aim was to infer the 'bias' of OV cells to respond to the object. This is analogous to inferring the bias of a coin after observing $r$ heads and $(n-r)$ tails in $n$ trials. The likelihood was calculated as

$$
p(D \mid y)=\frac{n !}{r !(n-r) !} y^{r}(1-y)^{n-r}
$$

where $y$ is the bias, $y \in[0,1]$. Next, we used a Cauchy likelihood (Supplementary Fig. 8b). The Cauchy distribution is a unimodal distribution like the Gaussian but has much fatter tails. We took this as a reasonable test of the sensitivity of the results to the shape of the likelihood function. In this case, we first calculated the likelihood given two parameters (the median ' $F R^{\prime}$ and the half-width ' $b$ ')

$$
p(D \mid F R, b)=\frac{1}{\pi} \frac{b}{\left(b^{2}+\left(d_{1}-F R\right)^{2}\right)} \times \frac{1}{\pi} \frac{b}{\left(b^{2}+\left(d_{2}-F R\right)^{2}\right)} \times \cdots \frac{1}{\pi} \frac{b}{\left(b^{2}+\left(d_{n}-F R\right)^{2}\right)}
$$

We then calculated the marginalized likelihood 


$$
p(D \mid F R)=\sum_{b} p(D \mid F R, b)
$$

630 The pattern of results from the Bayesian statistical analysis (Fig. 5) was similar (or stronger) when 631 changing the prior (Supplementary Fig. 7) and when changing the likelihood function (Supplementary

632 Fig. 8) confirming that the results in the main analysis were insensitive to the precise assumptions 633 made (a Gaussian likelihood and a uniform prior; Fig. 5).

634 Histology and reconstruction of recording positions.

635 The tetrodes were not moved after the final recording session. The mouse was given an overdose of 636 pentobarbital and was perfused intracardially with $9 \%$ saline and $4 \%$ formaldehyde. The brain was 637 extracted and stored in $4 \%$ formaldehyde. Frozen, $30-\mathrm{mm}$ sagittal sections were cut, mounted on glass, 638 and stained with cresyl violet (Nissl). The final position of the tip of each tetrode was identified on 639 photomicrographs obtained with an Axio Scan.Z1 microscope and Axio Vision software (Carl Zeiss) 640 (Supplementary Fig. 1).

641 Statistical tests. All statistical tests were two-sided. We used Kruskal-Wallis tests for variance analysis 642 between groups and Wilcoxon signed-rank tests for paired tests. Correlations were determined using 643 Pearson's product-moment correlation coefficients. No statistical methods were used to pre644 determine sample sizes but our sample sizes are similar to those reported in previous publications. The 645 study contained no randomization to experimental treatments and no blinding.

646 Data availability

647 Datasets supporting the findings of this paper are available upon request from the corresponding 648 authors.

\section{Code availability}

650 Custom code used in this paper is available upon request from the corresponding authors. 


\section{References}

1 Moser, E. I., Kropff, E. \& Moser, M. B. Place cells, grid cells, and the brain's spatial representation system. Annu Rev Neurosci 31, 69-89, doi:10.1146/annurev.neuro.31.061307.090723 (2008).

2 Moser, E. I., Moser, M. B. \& McNaughton, B. L. Spatial representation in the hippocampal formation: a history. Nat Neurosci 20, 1448-1464, doi:10.1038/nn.4653 (2017).

3 Fyhn, M., Molden, S., Witter, M. P., Moser, E. I. \& Moser, M. B. Spatial representation in the entorhinal cortex. Science 305, 1258-1264, doi:10.1126/science.1099901 (2004).

4 Hafting, T., Fyhn, M., Molden, S., Moser, M. B. \& Moser, E. I. Microstructure of a spatial map in the entorhinal cortex. Nature 436, 801-806, doi:10.1038/nature03721 (2005).

5 Boccara, C. N. et al. Grid cells in pre- and parasubiculum. Nature Neuroscience 13, 987-U112, doi:10.1038/nn.2602 (2010).

6 Taube, J. S., Muller, R. U. \& Ranck, J. B., Jr. Head-direction cells recorded from the postsubiculum in freely moving rats. I. Description and quantitative analysis. J Neurosci 10, 420-435 (1990).

7 Sargolini, F. et al. Conjunctive representation of position, direction, and velocity in entorhinal cortex. Science 312, 758-762, doi:10.1126/science.1125572 (2006).

8 Kropff, E., Carmichael, J. E., Moser, M. B. \& Moser, E. I. Speed cells in the medial entorhinal cortex. Nature 523, 419-424, doi:10.1038/nature14622 (2015).

9 Solstad, T., Boccara, C. N., Kropff, E., Moser, M. B. \& Moser, E. I. Representation of geometric borders in the entorhinal cortex. Science 322, 1865-1868, doi:10.1126/science.1166466 (2008).

10 Savelli, F., Yoganarasimha, D. \& Knierim, J. J. Influence of boundary removal on the spatial representations of the medial entorhinal cortex. Hippocampus 18, 1270-1282, doi:10.1002/hipo.20511 (2008).

11 O'Keefe, J. \& Dostrovsky, J. The hippocampus as a spatial map. Preliminary evidence from unit activity in the freely-moving rat. (1971).

12 O'Keefe, J. \& Nadel, L. The hippocampus as a cognitive map. (Clarendon Press; Oxford University Press, 1978).

13 Barry, C. et al. The boundary vector cell model of place cell firing and spatial memory. Rev Neurosci 17, 71-97, doi:10.1515/revneuro.2006.17.1-2.71 (2006).

14 Lever, C., Burton, S., Jeewajee, A., O'Keefe, J. \& Burgess, N. Boundary vector cells in the subiculum of the hippocampal formation. J Neurosci 29, 9771-9777, doi:10.1523/JNEUROSCI.1319-09.2009 (2009).

15 Bicanski, A. \& Burgess, N. Neuronal vector coding in spatial cognition. Nat Rev Neurosci 21, 453-470, doi:10.1038/s41583-020-0336-9 (2020).

16 Gothard, K. M., Skaggs, W. E., Moore, K. M. \& McNaughton, B. L. Binding of hippocampal CA1 neural activity to multiple reference frames in a landmark-based navigation task. The Journal of Neuroscience 16, 823, doi:10.1523/JNEUROSCI.16-02-00823.1996 (1996).

17 Rivard, B., Li, Y., Lenck-Santini, P.-P., Poucet, B. \& Muller, R. U. Representation of objects in space by two classes of hippocampal pyramidal cells. J Gen Physiol 124, 9-25, doi:10.1085/jgp.200409015 (2004).

18 Deshmukh, S. S. \& Knierim, J. J. Influence of local objects on hippocampal representations: Landmark vectors and memory. Hippocampus 23, 253-267, doi:10.1002/hipo.22101 (2013).

19 LaChance, P. A., Todd, T. P. \& Taube, J. S. A sense of space in postrhinal cortex. Science 365, 141-+, doi:ARTN eaax419210.1126/science.aax4192 (2019).

20 Wang, C. et al. Egocentric coding of external items in the lateral entorhinal cortex. Science 362, 945-+, doi:10.1126/science.aau4940 (2018).

21 Sarel, A., Finkelstein, A., Las, L. \& Ulanovsky, N. Vectorial representation of spatial goals in the hippocampus of bats. Science 355, 176-180, doi:10.1126/science.aak9589 (2017). 
22 Jercog, P. E. et al. Heading direction with respect to a reference point modulates place-cell activity. Nat Commun 10, 2333, doi:10.1038/s41467-019-10139-7 (2019).

23 Hoydal, O. A., Skytoen, E. R., Andersson, S. O., Moser, M. B. \& Moser, E. I. Object-vector coding in the medial entorhinal cortex. Nature 568, 400-404, doi:10.1038/s41586-019-10777 (2019).

24 Bayes. An Essay towards solving a Problem in the Doctrine of Chances: communicated by Mr. Price, in a Letter to John Canton, A. M. F. R. S. Philosophical transactions (Royal Society (Great Britain) : 1683) 53, 370 (1763).

25 Jeffreys, H. Theory of probability. 2nd ed. edn, (Clarendon Press, 1948). Jaynes, E. T. Probability theory : the logic of science. (Cambridge University Press, 2003). Gregory, P. C. Bayesian logical data analysis for the physical sciences : a comparative approach with Mathematica support. (Cambridge University Press, 2005).

28 Trotta, R. Bayes in the sky: Bayesian inference and model selection in cosmology. Contemporary Physics 49, 71-104, doi:10.1080/00107510802066753 (2008). Trotta, R. Bayesian Methods in Cosmology. (2017). Shadlen, M. N. \& Newsome, W. T. Noise, neural codes and cortical organization. Current Opinion in Neurobiology 4, 569-579, doi:https://doi.org/10.1016/0959-4388(94)90059-0 (1994).

31 Zhang, S.-J. et al. Optogenetic Dissection of Entorhinal-Hippocampal Functional Connectivity. Science 340, 1232627, doi:10.1126/science.1232627 (2013).

32 Buetfering, C., Allen, K. \& Monyer, H. Parvalbumin interneurons provide grid cell-driven recurrent inhibition in the medial entorhinal cortex. Nature Neuroscience 17, 710-718, doi:10.1038/nn.3696 (2014).

33 Diehl, G. W., Hon, O. J., Leutgeb, S. \& Leutgeb, J. K. Grid and Nongrid Cells in Medial Entorhinal Cortex Represent Spatial Location and Environmental Features with Complementary Coding Schemes. Neuron 94, 83-92.e86, doi:https://doi.org/10.1016/j.neuron.2017.03.004 (2017).

34 Hardcastle, K., Maheswaranathan, N., Ganguli, S. \& Giocomo, L. M. A Multiplexed, Heterogeneous, and Adaptive Code for Navigation in Medial Entorhinal Cortex. Neuron 94, 375-+, doi:10.1016/j.neuron.2017.03.025 (2017).

35 Miao, C. L., Cao, Q. C., Moser, M. B. \& Moser, E. I. Parvalbumin and Somatostatin Interneurons Control Different Space-Coding Networks in the Medial Entorhinal Cortex. Cell 171, 507-+, doi:10.1016/j.cell.2017.08.050 (2017).

36 McNaughton, B. L., Battaglia, F. P., Jensen, O., Moser, E. I. \& Moser, M.-B. Path integration and the neural basis of the 'cognitive map'. Nat Rev Neurosci 7, 663-678, doi:10.1038/nrn1932 (2006).

37 Schmitzer-Torbert, N., Jackson, J., Henze, D., Harris, K. \& Redish, A. D. Quantitative measures of cluster quality for use in extracellular recordings. Neuroscience 131, 1-11, doi:https://doi.org/10.1016/i.neuroscience.2004.09.066 (2005).

38 Skaggs, W., McNaughton, B., Gothard, K. \& Markus, E. An Information-Theoretic Approach to Deciphering the Hippocampal Code. Neural Inf. Process Syst. 5 (1997).

39 Barry, C. \& Burgess, N. To be a Grid Cell: Shuffling procedures for determining "Gridness". bioRxiv, 230250, doi:10.1101/230250 (2017).

40 Langston, R. F. et al. Development of the Spatial Representation System in the Rat. Science 328, 1576, doi:10.1126/science.1188210 (2010).

41 Wills, T. J., Cacucci, F., Burgess, N. \& Keefe, J. Development of the Hippocampal Cognitive Map in Preweanling Rats. Science 328, 1573, doi:10.1126/science.1188224 (2010).

42 Hartley, T., Burgess, N., Lever, C., Cacucci, F. \& O'Keefe, J. Modeling place fields in terms of the cortical inputs to the hippocampus. Hippocampus 10, 369-379, doi:https://doi.org/10.1002/1098-1063(2000)10:4<369::AID-HIPO3>3.0.CO;2-0 (2000). 


\section{Acknowledgments}

We thank $\varnothing$. Høydal for discussion and sharing code, J. Carpenter and B. Kanter for proofreading and L. Porta Mana for discussion of the field detection algorithm. We thank A.M. Amundsgård, N.Dagslott, K. Haugen, K. Jenssen, E. Kråkvik, I. Ulsaker-Janke and H. Waade for technical assistance. The work was supported by an RCN FRIPRO grant to M.-B.M. (grant number 300394), a Centre of Excellence scheme grant to M.-B.M. and E.I.M. and a National Infrastructure grant to E.I.M. and M.-B.M. from the Research Council of Norway (Centre of Neural Computation, grant number 223262; NORBRAIN, grant number 295721), the Kavli Foundation (M.-B.M. and E.I.M.), and a direct contribution to M.-B.M. and E.I.M. from the Ministry of Education and Research of Norway.

\section{Author Contributions}

S.O.A., M.-B.M. and E.I.M. designed experiments; S.O.A. performed experiments and performed all analyses; S.O.A., M.-B.M. and E.I.M. interpreted data; S.O.A. wrote the paper, with assistance from E.I.M. and M.-B.M.; E.I.M. and M.-B.M. supervised the project. 
a
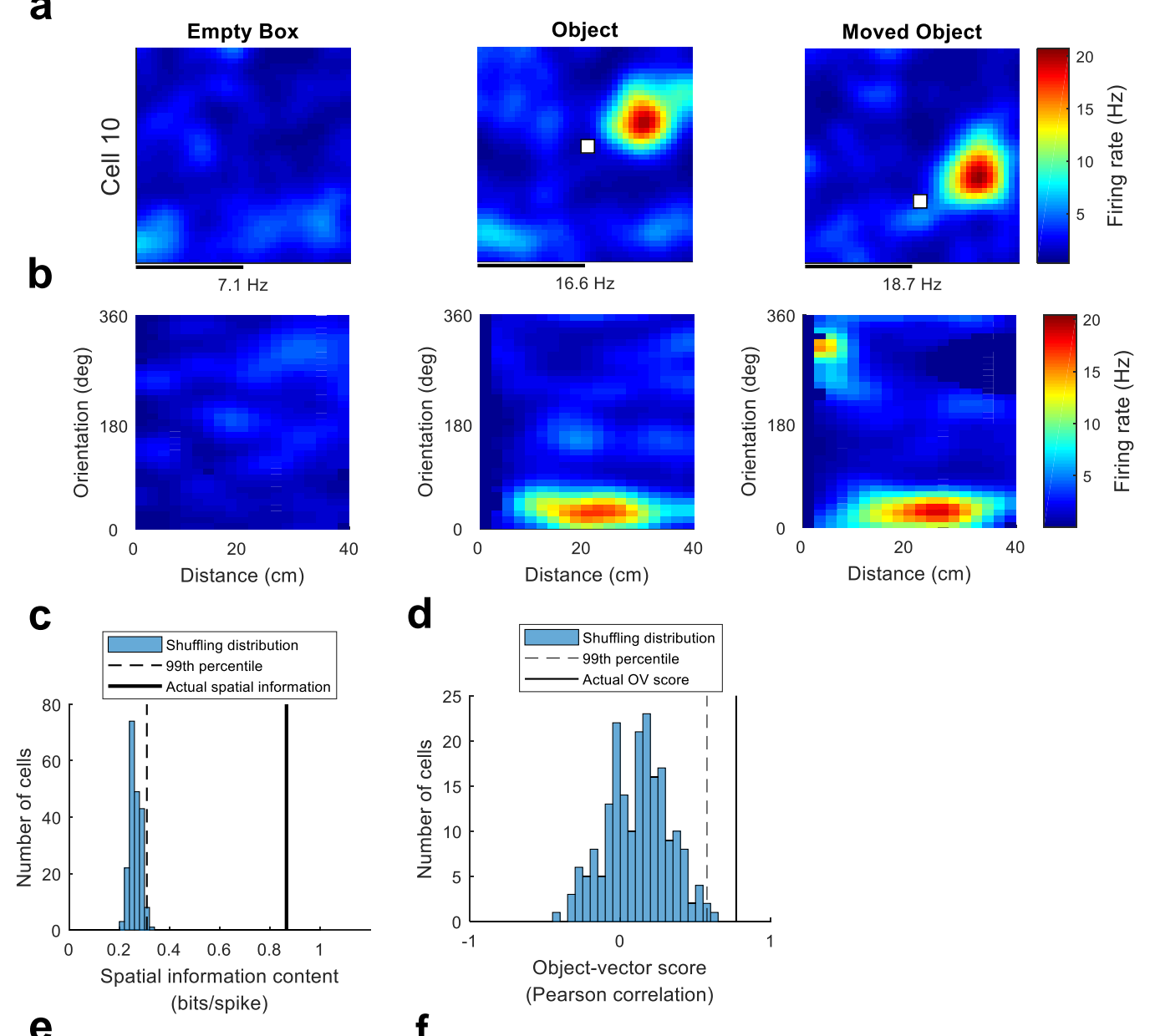

e

f
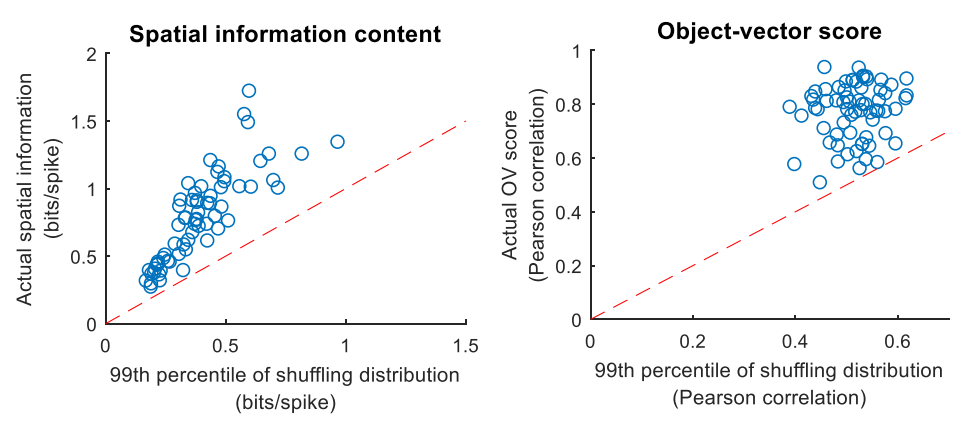

Figure 1. Identification of object-vector (OV) cells. a, Rate maps for example cell 10 from the three trials used to identify it as an OV cell. In the first trial, the environment is empty ('Empty Box'). In the second trial, a free-standing object made of Duplo bricks is present in the environment ('Object'). In the third trial, the same object is moved to a new location ('Moved Object'). Rate maps show colourcoded firing rate in $\mathrm{Hz}$ as a function of the animal's position. White squares mark the object location. Peak firing rate $(\mathrm{Hz})$ is indicated below each map. Scale bar represents $40 \mathrm{~cm}$. The example cell fires in a specific distance and direction away from the object, the defining behaviour of OV cells. b, Objectcentred rate maps (referred to as 'vector maps' in ref 23), displaying firing rate $(\mathrm{Hz})$ as a function of the animal's distance $(\mathrm{cm}$ ) and orientation (degrees) to the object. The maps are from the 'Empty Box', 'Object' and 'Moved Object' trials for the cell shown in panel a. For an OV cell, we expect the maps from the 'Object' and 'Moved Object' trials to be similar. c, Shuffling distribution of spatial information content for Example Cell 10 (same cell as in previous panels). The cell's spike timestamps were randomly shifted along the animal's trajectory $(n=200$ permutations). For each shuffled cell, we 
calculated the spatial information content, which quantifies how informative the spikes are about the animal's position. The actual spatial information content of the cell is far above the 99th percentile of the shuffling distribution. Spatial information contents were calculated using data from the 'Object' trial. $\mathbf{d}$, Shuffling distribution of OV scores for Example Cell 10. After performing shuffling (as in the previous panel) we calculated the OV score for each shuffled cell. The OV score is the Pearson correlation between the object-centred maps shown in panel b from the 'Object' and 'Moved Object' trials. The actual OV score of the cell exceeds the 99th percentile of the shuffling distribution. e, Scatterplot showing the actual spatial information content (bits/spike) of OV cells, compared to the threshold value obtained from each cell's shuffling distribution. The threshold was the 99th percentile of the shuffling distribution. All data points fall above the diagonal because, by definition, OV cells need to pass the spatial information criterion. The spatial information content was calculated on the 'Object' trial (Fig. 1a). f, Scatterplot showing the actual OV score of OV cells, compared to the threshold value obtained from each cell's shuffling distribution. The threshold was the 99th percentile of the shuffling distribution. All data points fall above the diagonal because, by definition, OV cells need to pass the OV score criterion. The OV score is the Pearson correlation between the object-centered maps in Figure $1 \mathrm{~b}$ from the 'Object' and 'Moved Object' trials. 
a

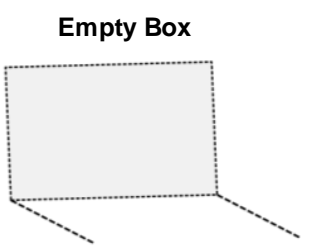

2D object

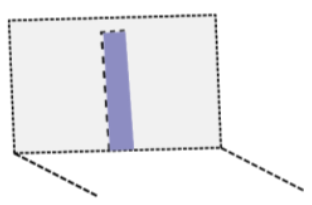

b

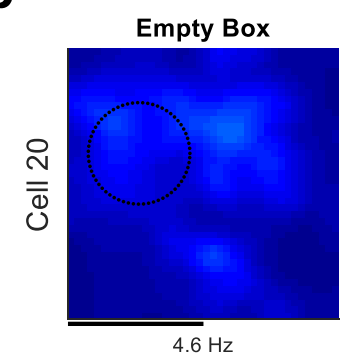

C
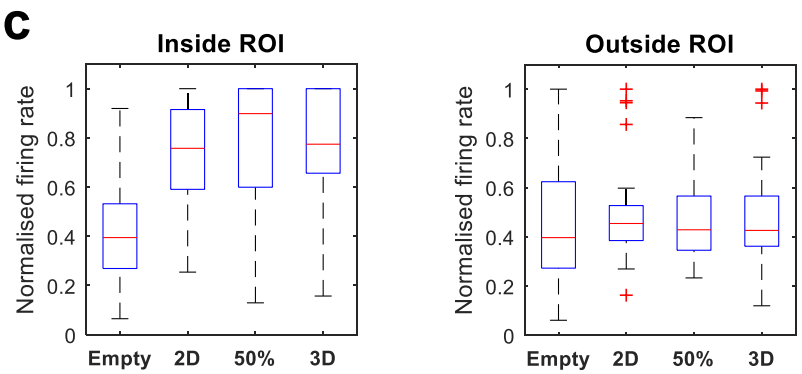

2D
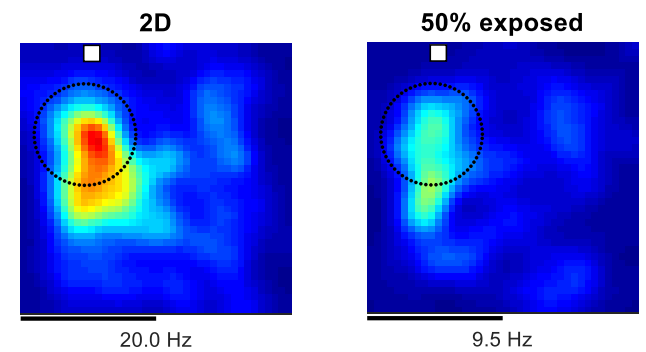

3D object
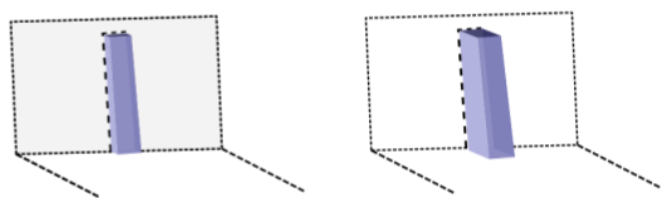

3D

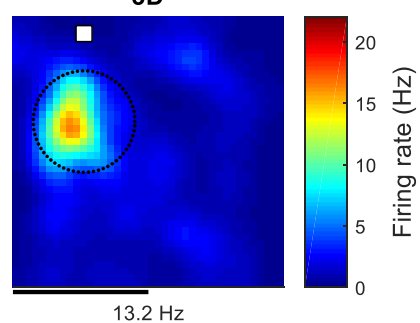

d

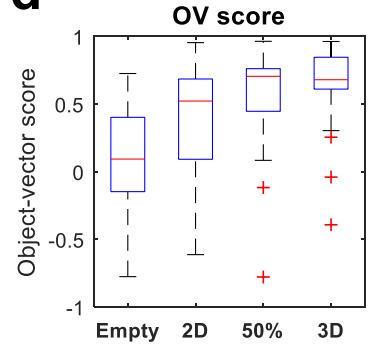

Figure 2. Object-vector cells respond to 2-dimensional objects. a, Experimental design with four different trials: mice foraged in an environment with either an object absent ('Empty Box') or present ('2D', '50\%', '3D'). In the three object trials, we varied the amount of the object's volume exposed to the animal. In the 2D trial, the visible part of the object was a flat 2D surface, with the rest completely embedded into the wall. In the middle trial, the object was partially embedded into the wall so that $50 \%$ of its volume was exposed. In the 3D trial, the full volume of the object was exposed. c, Colour-coded rate maps from example OV cell that responded as strongly to 2D surfaces as to 3D objects. Rate maps show colour-coded firing rate in $\mathrm{Hz}$ as a function of the animal's position. The white square marks the object location. The dotted circle marks the ROI in which we expected the cell to fire based on its vector coordinates. The vector coordinates of the cell were found by applying the algorithm from Figure 1 to the 'Object' trial in which we originally identified the cell as an OV cell. Peak firing rate $(\mathrm{Hz})$ is indicated below each map. Scale bar, $40 \mathrm{~cm}$. d, Box-and-whisker plots of normalised firing rates $(\mathrm{Hz})$ of object-vector cells $(n=30)$ as a function of object dimensionality. Firing rates were normalised to the maximum data point across all 8 data points observed for each cell ( 4 experimental conditions $\times$ inside/outside ROI) so that the maximum rate is 1. The middle line (in red) on each box indicates the median, while the bottom and top lines (in blue) indicate the lower and upper quartiles, respectively. Whiskers show the range of data in each condition. Red crosses show outliers that lie more than 1.5 times outside the interquartile range. The firing rate was measured either inside (left) or outside the ROI (right). e, Box-and-whisker plot of object-vector scores (ranging from -1 to 1 ) as a function of object dimensionality. The object-vector score is equivalent to the Pearson correlation between pairs of object-centred rate maps (see Method for details). Here, the reference rate map is from the 'Object' trial originally used to identify the cell. 
a

Empty Box

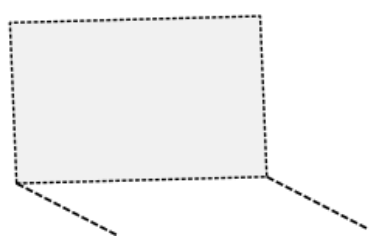

b

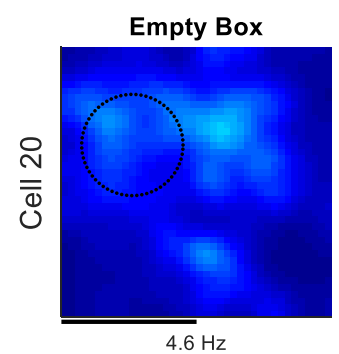

C

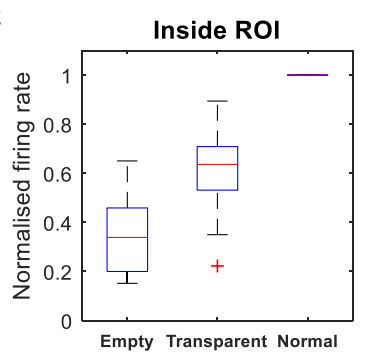

Transparent object (2D)

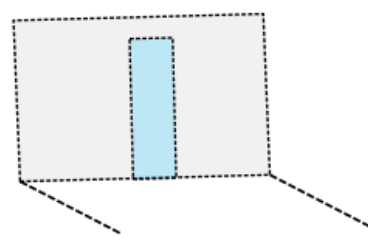

Transparent

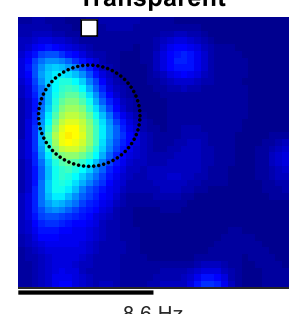

$8.6 \mathrm{~Hz}$

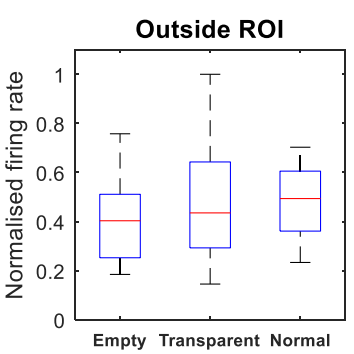

Standard object (3D)

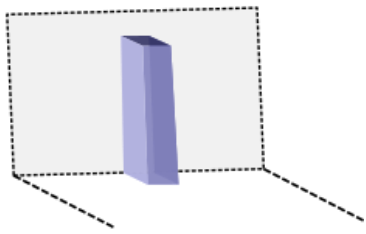

Normal
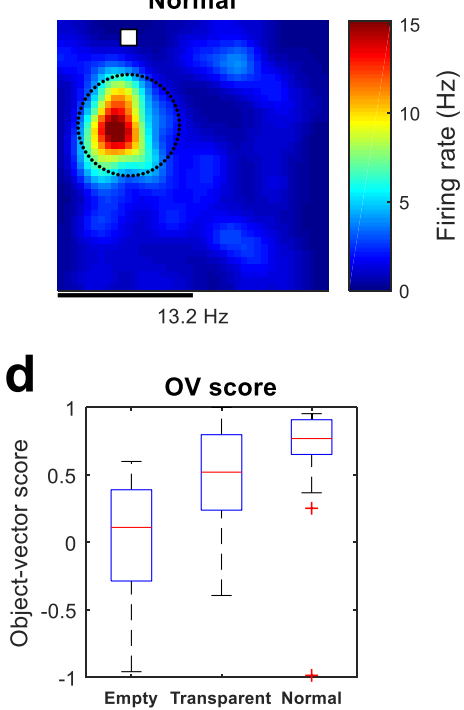

Figure 3. Object-vector cells respond to transparent objects. a, Experimental design with three different trials: either the box was empty, had a transparent object present, or a standard object present (a Duplo tower). $\mathbf{b}$, Rate maps from example OV cell that responded strongly to the transparent object. Rate map conventions are as in Figure 2. Scale bar, $40 \mathrm{~cm}$. c, Box-and-whisker plots of normalised firing rates $(\mathrm{Hz})$ of OV cells $(n=14)$ as a function of discreteness of the object, inside (left) or outside the ROI (right). d, OV score as a function of object type. Conventions for boxand-whisker plots are as in Figure 2. 
a
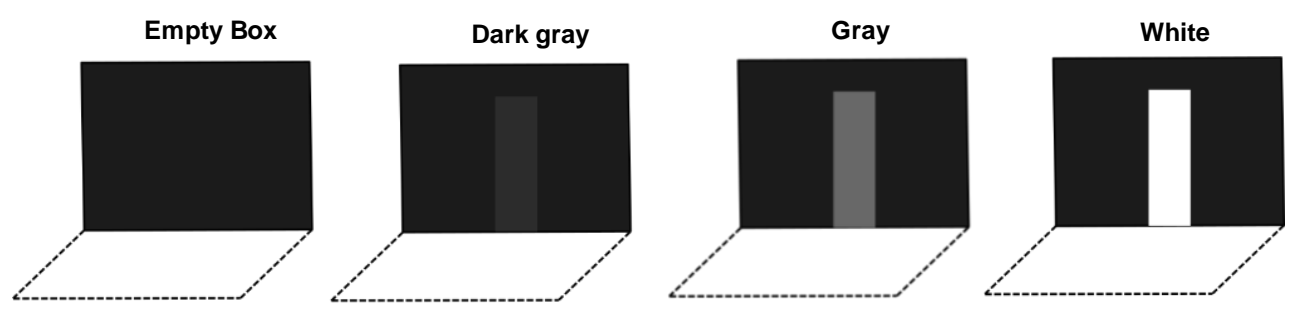

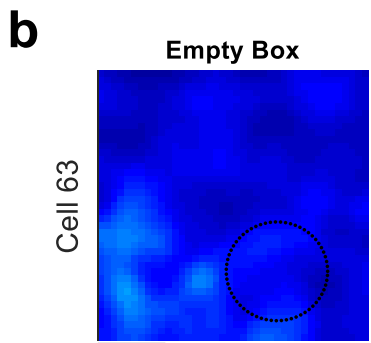

$2.9 \mathrm{~Hz}$

C

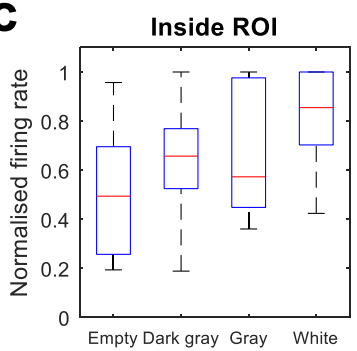

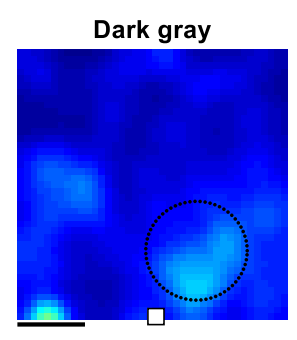

$3.4 \mathrm{~Hz}$

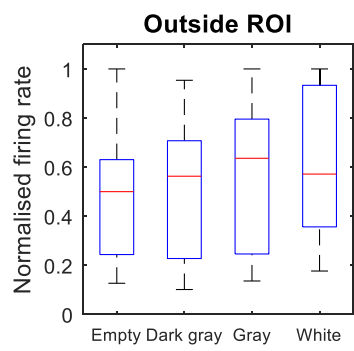

Gray

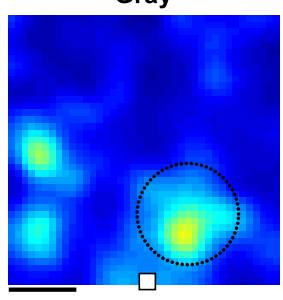

$5.5 \mathrm{~Hz}$
White

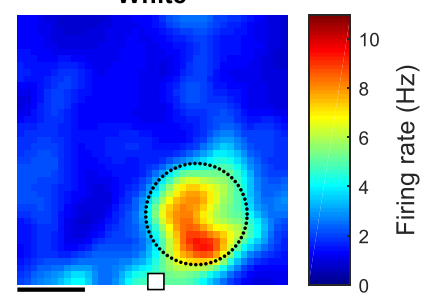

$8.0 \mathrm{~Hz}$

Figure 4. Responses of object-vector cells to visual contrasts. a, Experimental design with four different trials: either the box was empty or had a visual contrast present. In the three trials with a visual contrast, we varied the object's contrast from dark grey, to grey, to white $(10 \%, 60 \%$ and $100 \%$ whiteness, respectively). b. Example rate maps from OV cell clearly increasing its firing rate as a function of visual contrast. Rate map conventions are as in Figure 2 . Scale bar, $20 \mathrm{~cm}$. c, Box-andwhisker plots of normalised firing rates $(\mathrm{Hz})$ of OV cells $(n=14)$ as a function of the object's visual contrast. The firing rate was measured either inside (left) or outside the ROI (right). d, Box-and whisker plot of OV scores as a function of visual contrast. Conventions for box-and-whisker plots are as in Figure 2. 
a

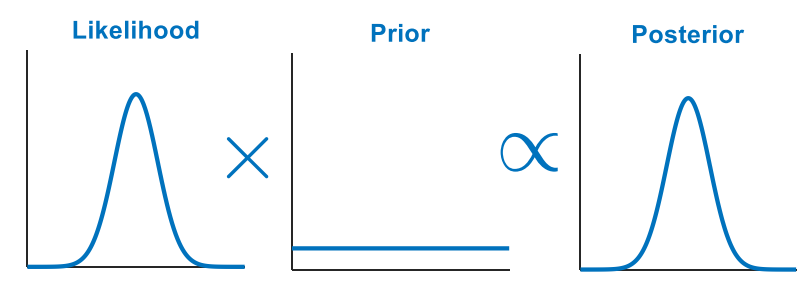

b

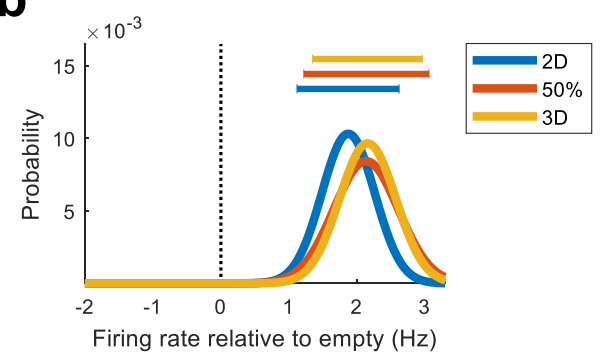

C

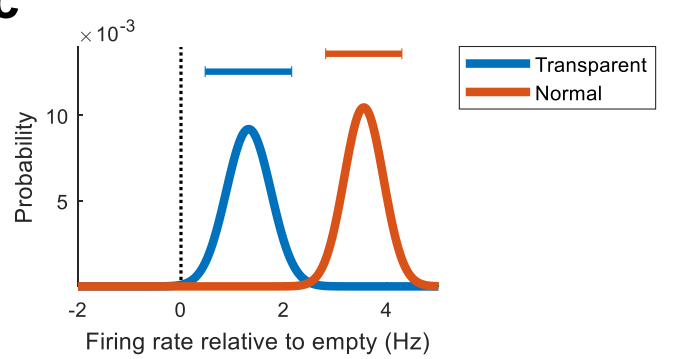

d

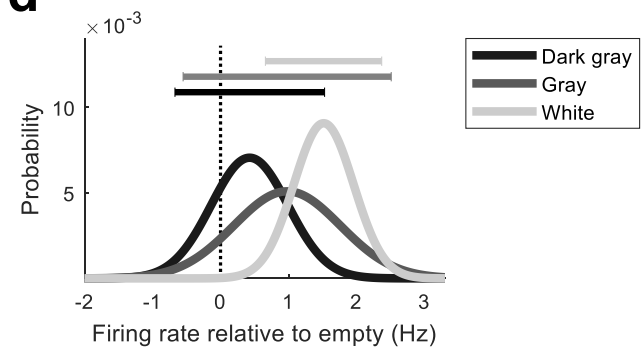

Figure 5. Posterior probability distributions of the average firing rates of OV cells to each object or feature. a, Visualisation of Bayesian inference. Bayes' theorem multiplies the likelihood with a prior to give the posterior distribution summarising our state of knowledge. The likelihood shows which average firing rates (FRs) best explain the data obtained. The prior gives the probabilities of different firing rates before seeing the data. The resulting posterior shows the probability that OV cells respond with any average FR to the object. For the distributions in Figure 5, we assigned a Gaussian likelihood and assumed a uniform prior (see Method for details). We verified that our conclusions were insensitive to these assumptions (Supplementary Fig. 7 and 8). b, Posterior distributions showing the probability that OV cells respond with any average FR to each object. The plots correspond to Figure 2 and describe the results for the 2D surface (blue curve), the partially embedded object (red curve) and the 3D object (yellow curve). The data are the firing rates of OV cells inside the region in which we expect each cell to fire based on its vector coordinates. The firing rate in the same region in the 'Empty Box' trial has been subtracted. This means that probability on the right (left) of $0 \mathrm{~Hz}$ should be interpreted as a positive (negative) response to the object. $0 \mathrm{~Hz}$ is marked by the stippled line. The intervals at the top represent the 'credible region', which is the smallest possible region containing $95 \%$ of the probability mass. The credible region can be interpreted as "there is a $95 \%$ probability that OV cells respond with an average FR between these bounds". c, Same as in the previous panel but probability distributions corresponding to Figure 3 describing the results for the transparent surface (blue curve) and normal 3D tower (red curve). d, Same as in the previous panels but probability distributions corresponding to Figure 4 describing the results for the dark gray, gray and white contrast. Note that while all curves peak at positive values, the right-shift of the curves increases gradually with increasing visual contrast. 
bioRxiv preprint doi: https://doi org/10.1101/2021 06.03.446869; this version posted June 3, 2021. The copyright holder for this preprint

(which was not certified by peer review) is the author/funder, who has granted bioRxiv a license to display the preprint in perpetuity. It is

made available under aCC-BY-NC-ND 4.0 International license Supplementary Figure 1

88162 , left MEC

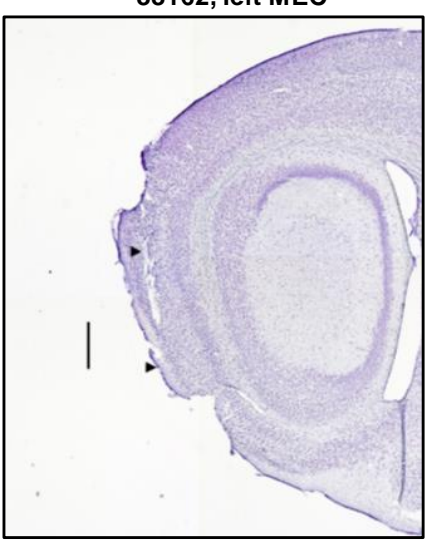

83442, left MEC

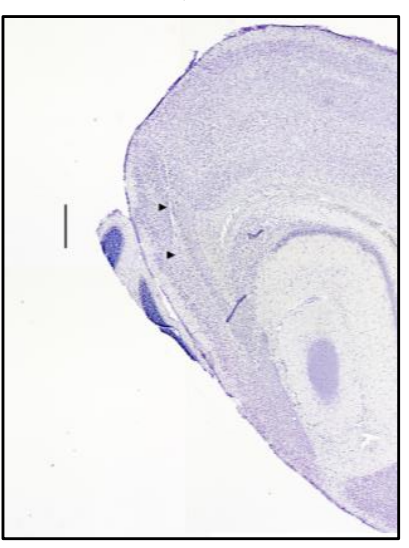

82651, left MEC

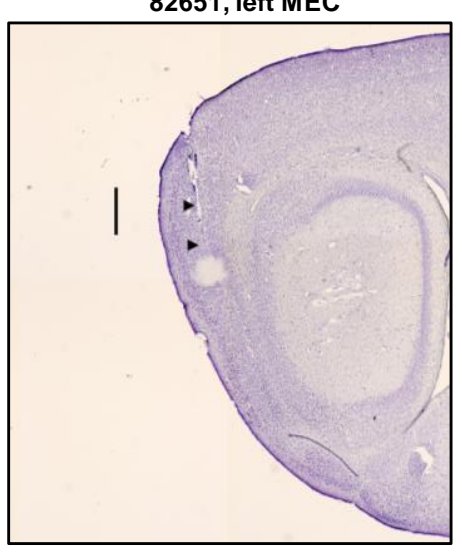

87685, left MEC

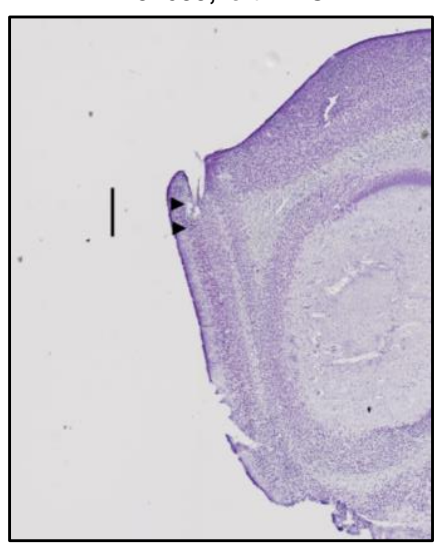

82667, left MEC

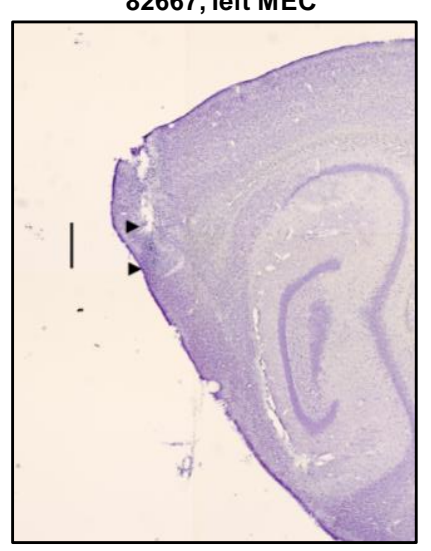

85669, right MEC

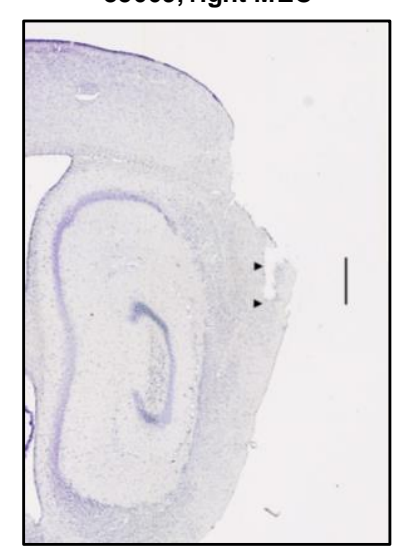

87684, left MEC

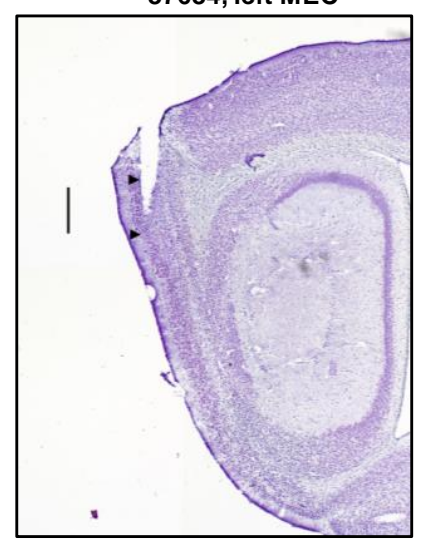

Supplementary Figure 1. Recording locations in the MEC. Nissl-stained sagittal brain sections showing tetrode locations for the 7 mice used in the experiments. Mouse identifier (ID) numbers and brain hemisphere are indicated above each section. Pairs of black arrowheads indicate the dorsoventral range of recording locations in which OV cells were found. 
a

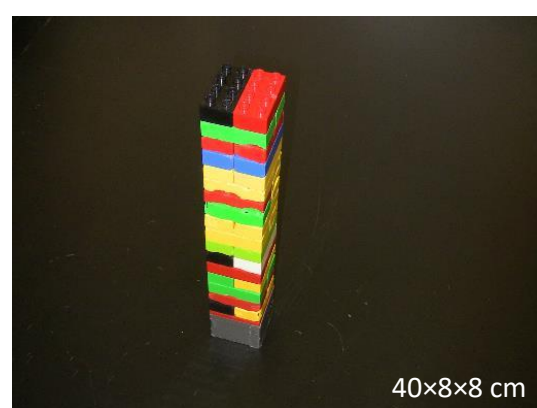

\section{b}

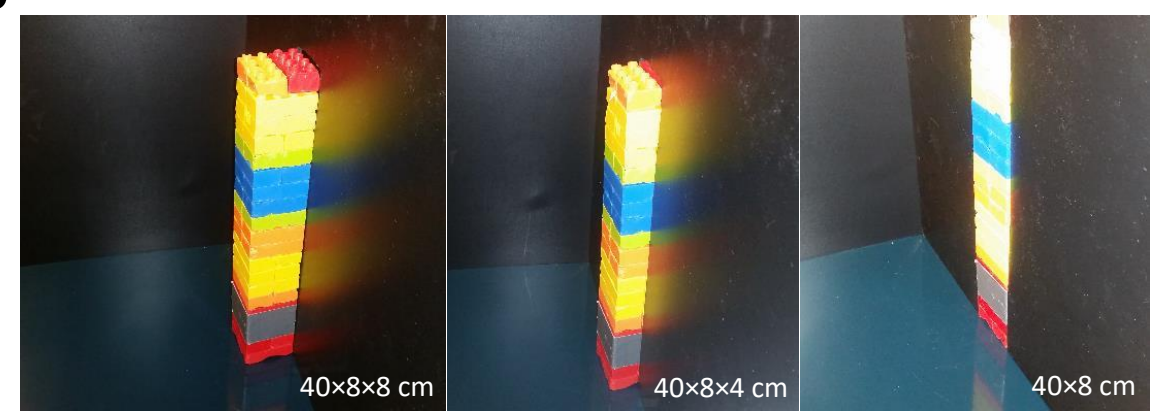

C

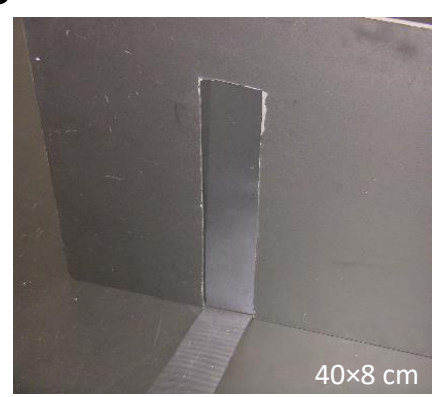

d

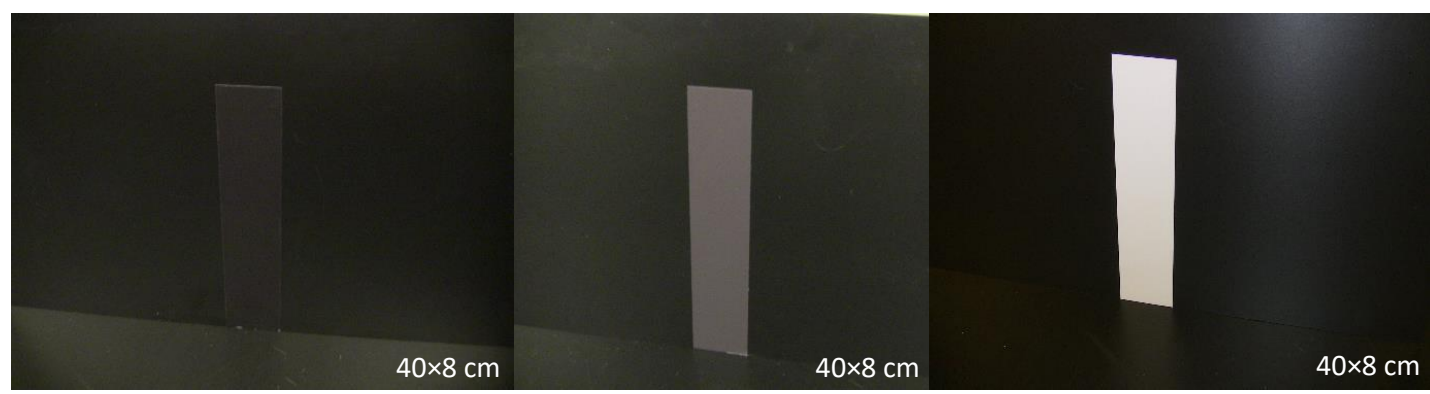

Supplementary Figure 2. Objects used in the experiments. a, Tower-like object made of Duplo used in the 'Object' and 'Moved Object' trials (Fig. 1a, middle and right) in order to identify OV cells. The object' size was $40 \times 8 \times 8 \mathrm{~cm}^{3}$ (height, length, width). b, Objects used in the 2D/3D experiment (Fig. 2). Three configurations were tested: in one (left), the full 3D shape of the object was inside the arena, exposing $100 \%$ of its volume. In a second configuration (middle), the object was partially embedded into the wall, exposing $50 \%$ of its volume. In the third configuration (right), the object was fully embedded into the arena wall, appearing as a visually distinct segment of the otherwise continuous arena wall. c, Transparent 2D surface used in the experiment reported in Fig. 3. A transparent film was present on the outside of a $40 \times 8 \mathrm{~cm}^{2}$ hole in the arena wall. Behind the wall and the opening, the animal could see a uniform surface formed by dark blue curtains surrounding the test arena (located $\sim 1 \mathrm{~m}$ away from the arena walls). $\mathrm{d}$, Visual contrasts used in the experiment reported in Fig. 4 . The visual contrast was a $40 \times 8 \mathrm{~cm}^{2}$ band of self-adhesive tape printed on the wall, with varying levels of whiteness $(10 \%, 60 \%$ and $100 \%$, respectively). 
a

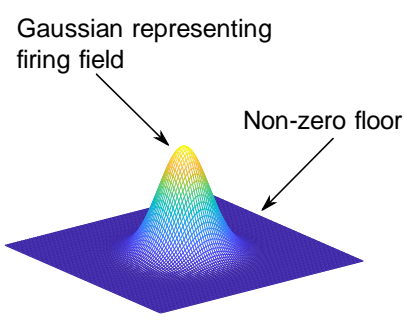

b
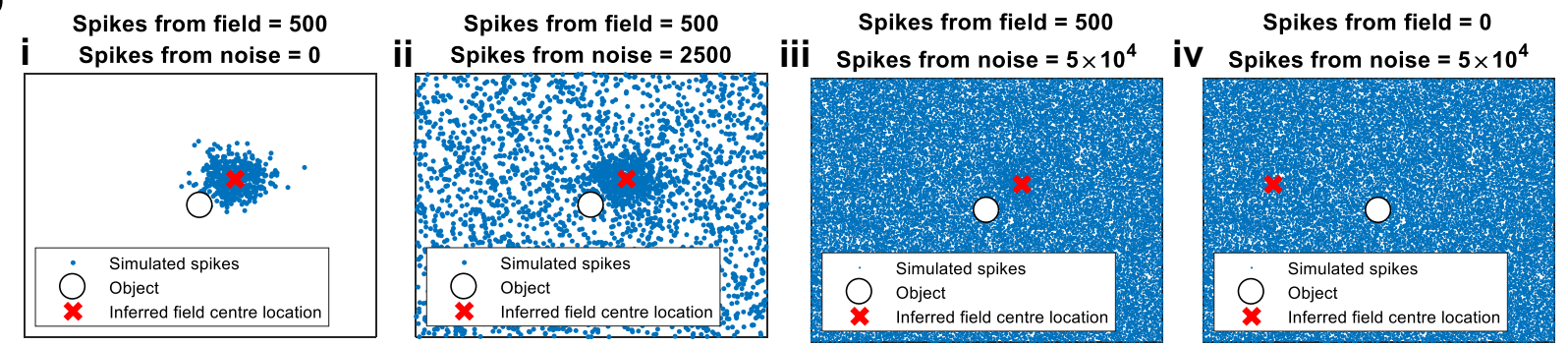
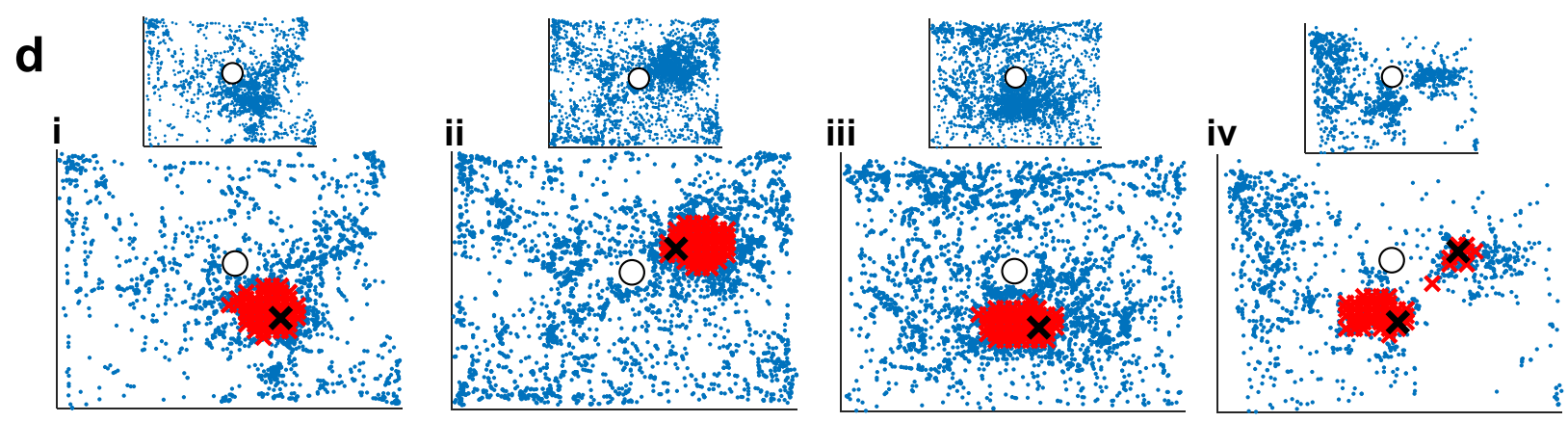

Supplementary Figure 3. Field detection algorithm. a, Firing fields were detected by an algorithm that models the spike data as a mixture of two processes. The first process is a Gaussian representing the cell's firing field. The second process is a uniform floor with non-zero probability (a Gaussian with infinite variance). This represents the fact that the cell might spike anywhere in the environment, regardless of its spatial firing pattern. The second process is the one responsible for clearing up noise. b, Representative examples of field detection on simulated data. In the first three examples, the number of spikes drawn from the field is 500 , while the number of spikes drawn from noise is varied between 0 (i), 2500 (ii) and $5 \times 10^{4}$ (iii). The algorithm identifies the correct location of the field in all cases. When only noise is present, as in the last example ( 0 spikes from the field, $5 \times 10^{4}$ spikes from noise), the algorithm chooses a random location (iv). c, Performance of algorithm with error in Euclidian distance $(\mathrm{cm})$ as a function of the number of spikes drawn from noise ( $\mathrm{x}$-axis) and the number of spikes drawn from the field (colored curves). When the number of spikes in the field is 500 or 250, perfect performance is achieved across all noise levels (mean error $0 \mathrm{~cm}$ ). When the number of spikes in the field is 125, the mean error is less than $2.5 \mathrm{~cm}$ for all noise levels except the largest one (mean error $11.8 \mathrm{~cm}$ ). The error is large only if the field contains 50 spikes or less. Note that 500 spikes from the field is the same as in examples from panel $b$, i-iii. The data points are means with 50 repetitions for each condition. The dotted line indicates chance level. $d$, Representative examples of field detection on real data. Red crosses show the $x, y$ coordinates with the 50 largest probability values in the algorithm's probability distribution for the fields. Black crosses show the $x, y$ coordinates with the maximum probability for each local cluster. Examples are from OV cells with one field (i-iii) and an OV cell with two fields (iv). The number of fields was determined by the number of local maxima in the probability distribution. Insets show path plots before field detection for clearer visualisation. 

made available under aCC-BY-NC-ND 4.0 International licenş Supplementary Figure 5

\section{2}
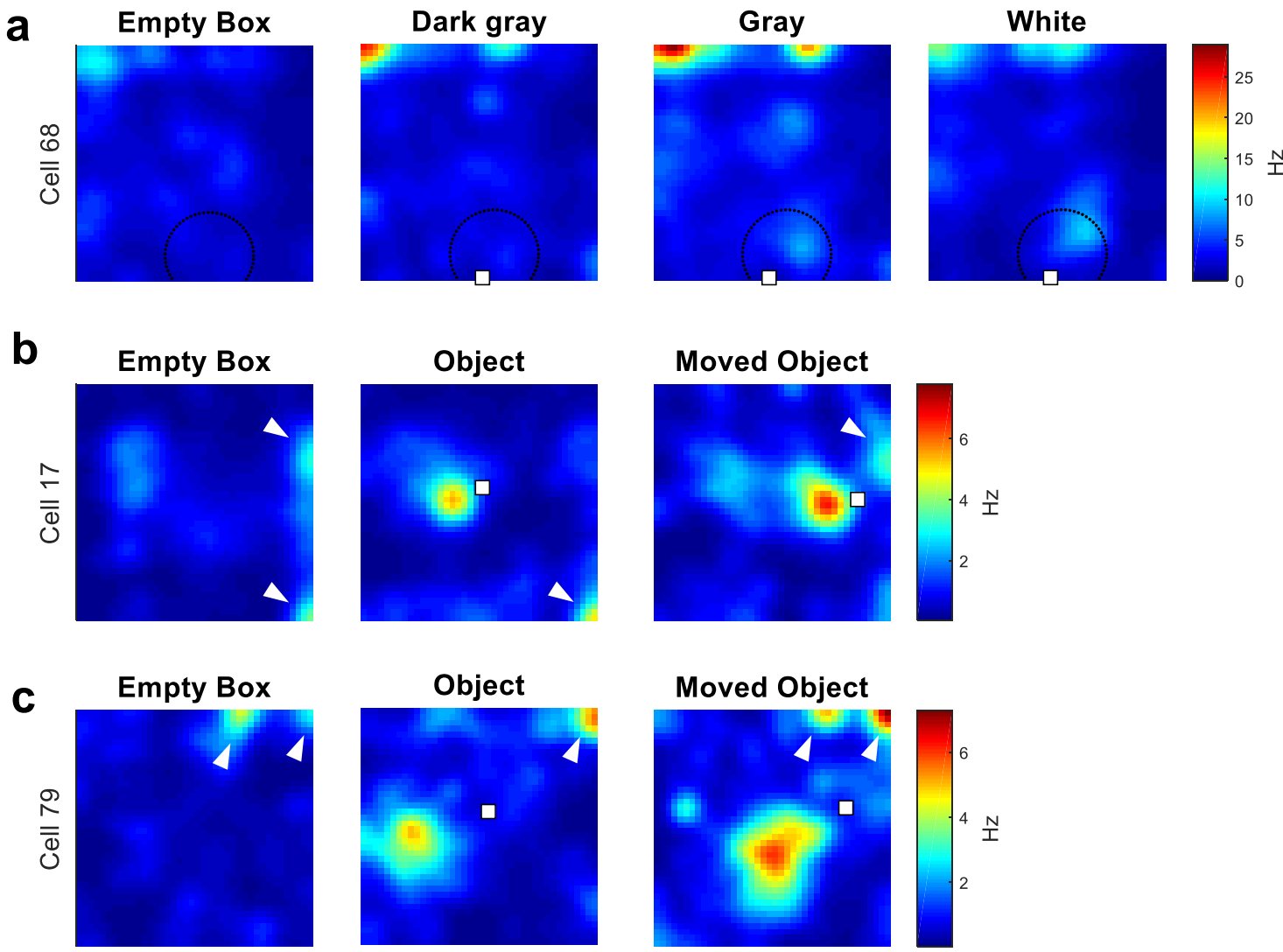

Supplementary Figure 5. Examples of OV cells with weak firing fields, only some of which are induced by the experimental object. a, Example of an OV cell in the contrast experiment (Fig. 4) where the change in contrast of the experimental object elicits a weak increase in firing rate that is difficult to detect by eye. Rate maps show colour-coded firing rate in $\mathrm{Hz}$ as a function of the animal's position. The white square marks the object location. The dotted circle marks the ROI in which we expected the cell to fire based on its vector coordinates. The vector coordinates of the cell were found by applying the algorithm described in Supplementary Fig. 3 to the 'Object' trial in which we originally identified the cell as an OV cell (Fig. 1a, middle). b, Example of an OV cell with weak firing fields that are not induced by the presence of the object. White arrows indicate the weak firing fields. Given the results from Fig. 4, such weak firing fields might reflect responses to edges, corners, shadows or other types of contrast (see Discussion). Rate map conventions are as in panel a. c, Further example of an OV cell with weak firing fields that are not induced by the presence of the object. White arrows indicate the weak firing fields. Rate map conventions are as in panel a. It is notable that in both panels $b$ and $c$ several of the weak firing fields line up with the corners of the recording box. Also note that in both panels, one weak firing field disappears when introducing the object (compare 'Empty Box' and 'Object'), raising the possibility that weak firing fields become inhibited by more salient objects when these are present in the environment. 


\section{a}

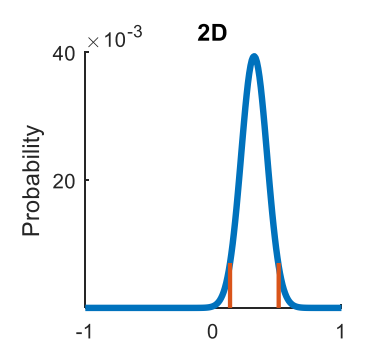

b

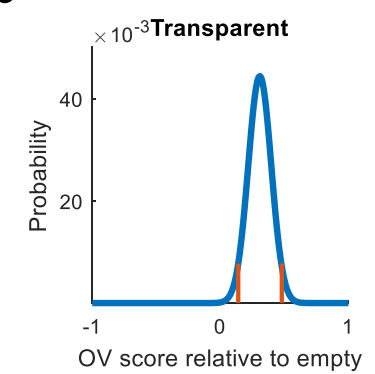

C

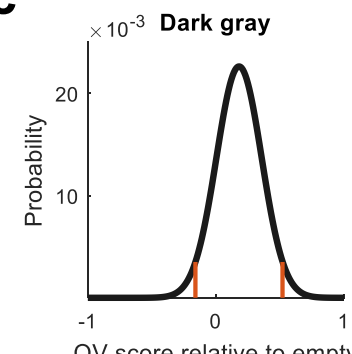

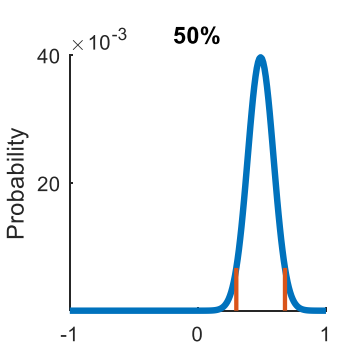

OV score relative to empty
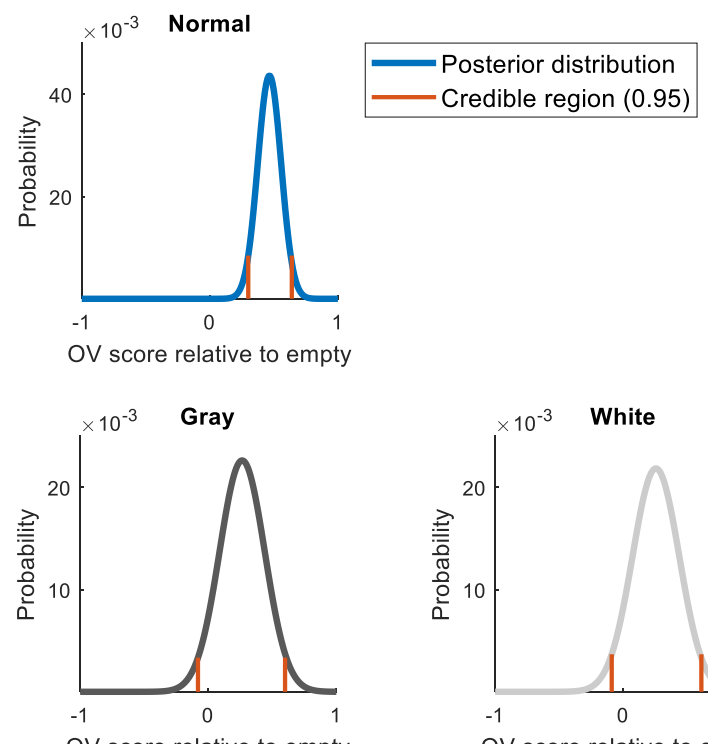

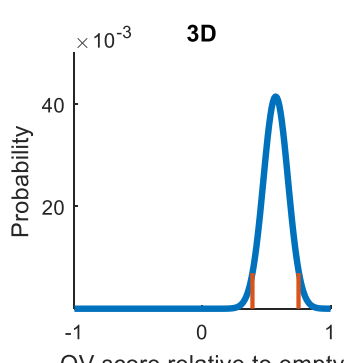

OV score relative to empty

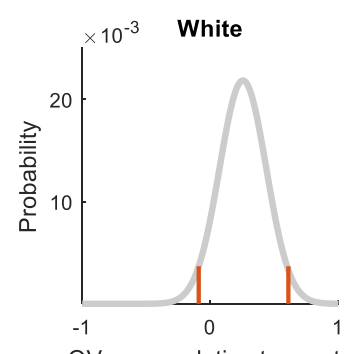

Posterior distribution

Credible region (0.95) d

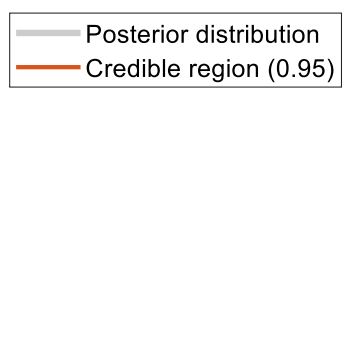

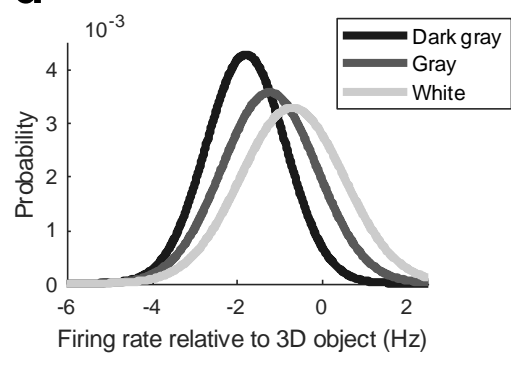

Supplementary Figure 6. Posterior distributions of the increase in OV score to each object or feature. Panels are similar to Fig. 5 but use the OV score rather than the firing rate as input to the Bayesian inference. The OV score in the 'Empty Box' trial has been subtracted so that probability on the right (left) of 0 should be interpreted as a positive (negative) response to the object or feature. Orange bounds represent the credible region, which is the smallest possible region containing $95 \%$ of the probability mass. The credible region can be interpreted as "there is a 95\% probability that the increase in the OV score lies between these bounds". a, Posterior distributions show the probability of the amount of increase in the OV score. The plots correspond to Fig. $2 \mathrm{~d}$ and show the results for the $2 \mathrm{D}$ surface (left), the partially embedded object (middle) and the 3D object (right). $\mathbf{b}$, Same as in panel a but posterior distributions from the transparent-object experiment in Fig. $3 \mathrm{~d}$. The results are shown for the transparent object in the wall (left) and the freestanding 3D object (right). c, Same as in panel a but posterior distributions from the contrast experiment in Fig. 4d. The results are shown for the dark gray contrast (left), the gray contrast (middle) and the white contrast (right). d, Probability distributions from the contrast experiment overlaid, plotting the probability of different firing rate changes $(\mathrm{Hz})$ relative to the 3D object. The panel is analogous to Fig. $5 \mathrm{e}$ but uses the 3D object rather than the 'Empty Box' trial as reference. Because all contrasts produce weaker activity in OV cells than the $3 \mathrm{D}$ object, all probability distributions lie to the left of the origin. The overall trend (increasing probability towards more positive firing rates as a function of contrast) is the same. The trial with the 3D object was the 'Moved Object' trial. 
a

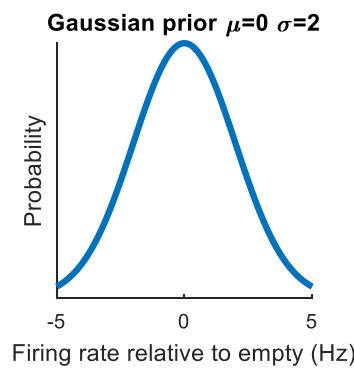

b

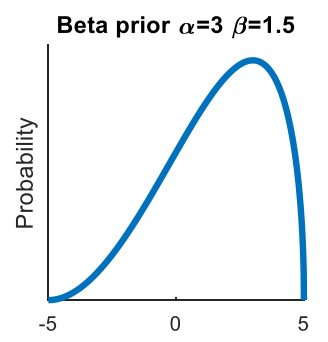

Firing rate relative to empty $(\mathrm{Hz})$

C

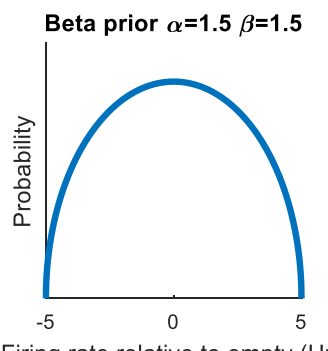

Firing rate relative to empty $(\mathrm{Hz})$
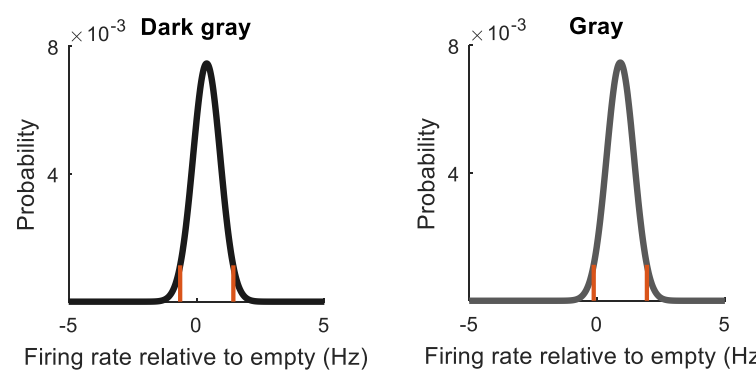

Firing rate relative to empty $(\mathrm{Hz})$
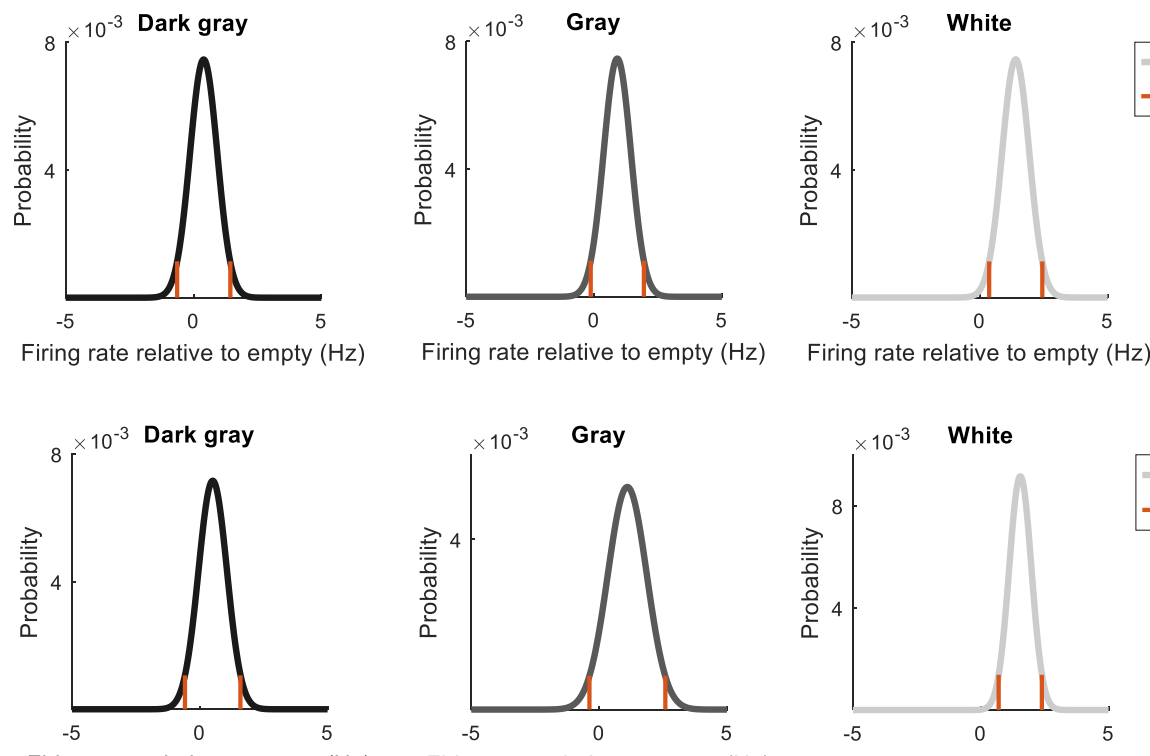

Firing rate relative to empty $(\mathrm{Hz})$

Firing rate relative to empty $(\mathrm{Hz})$

Firing rate relative to empty $(\mathrm{Hz})$
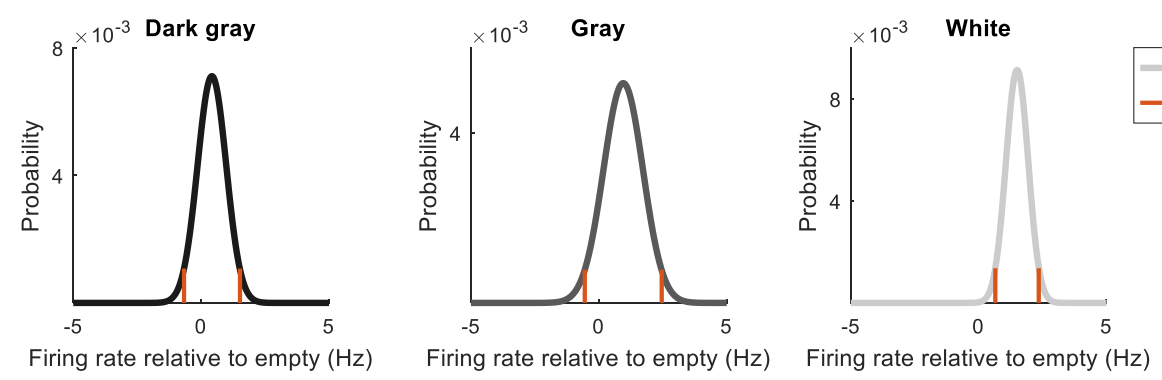

Supplementary Figure 7. Sensitivity of results to different priors. a, Posterior distributions, as in Figure $5 \mathrm{~d}$, showing the probability that OV cells respond with any average firing rate to each visual contrast. Instead of a uniform prior, we use a Gaussian prior peaked at 0 with a standard deviation of 2. The results from choosing this prior are effectively identical to the results in Fig. $5 \mathrm{~d}$. b, Same as in panel a but using a beta prior with parameters alpha $=3$, beta $=1.5$ in order to bias firing rates towards positive values. This intuitively corresponds to prior information that OV cells are more likely to respond with a higher firing rate when an object is present in the environment, pointing to this prior as a possible alternative to the more conservative uniform prior chosen in this study. The three panels to the right show that the results from choosing the prior in $b$ are effectively identical to the results in Fig. 5 d. c, Same as in panel a but using a beta prior with parameters alpha=1.5, beta=1.5 to admit a wide range of possible values but discount strong changes in FR. The results from choosing this prior are effectively identical to the results in Figure $5 \mathrm{~d}$. Overall, the results from panels $\mathrm{a}, \mathrm{b}$ and $\mathrm{c}$ confirm that the amount of data we have obtained is sufficient because any reasonable prior gives identical results. 
a

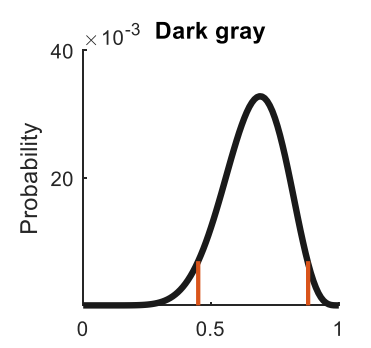

b

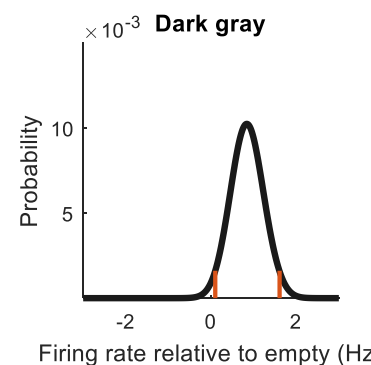

\section{Binomial likelihood}
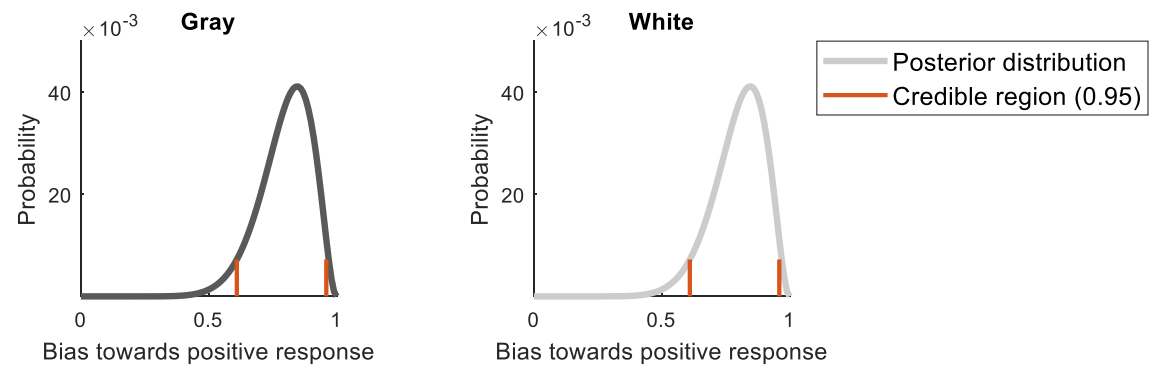

\section{Cauchy likelihood}
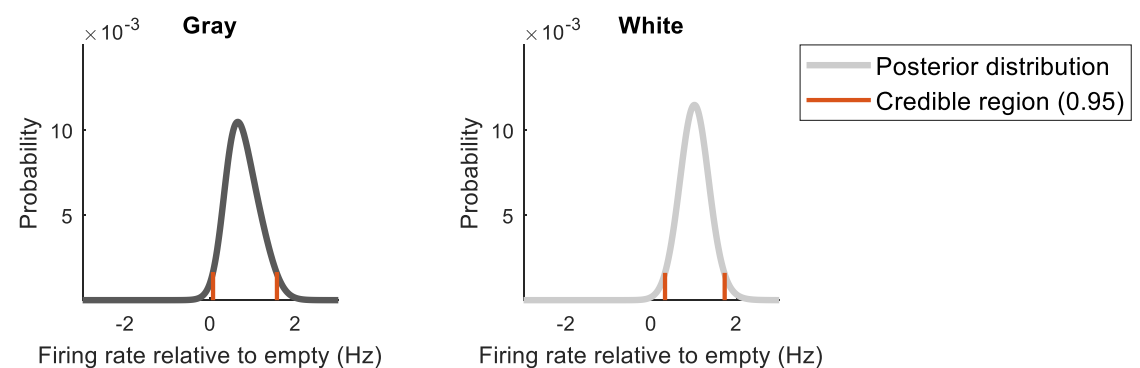

Supplementary Figure 8. Sensitivity of results to different likelihoods. a, Bayesian inference using a binomial likelihood instead of a Gaussian likelihood. Here, we have binarized the data into either response $(x=1)$ or no response $(x=-1)$ by applying the sign function to every data point (which gives $x=1$ if $F R$ is $>0 \mathrm{~Hz}$ and $x=-1$ if $F R$ is $<0 \mathrm{~Hz}$ ). This divides the data into $r$ successes and $(N-r)$ failures. $N$ is the total number of OV cells we have data from, $r$ is the number of OV cells that respond with a firing rate $>0 \mathrm{~Hz}$ and $\mathrm{N}-\mathrm{r}$ is the number of $\mathrm{OV}$ cells that respond with a firing rate $<0 \mathrm{~Hz}$. We then infer the 'bias' of OV cells to respond to the object or feature. Note that this is equivalent to inferring the bias of a coin after tossing the coin $\mathrm{N}$ times, observing $\mathrm{r}$ heads and $\mathrm{N}-\mathrm{r}$ tails. The posterior distributions of the bias of OV cells are shown for the dark gray contrast (left), the gray contrast (middle) and the white contrast (right). Note that as the visual contrast increases, the bias towards a positive response increases, consistent with the results presented in Fig. $5 d$ and e. b, Bayesian inference using a Cauchy likelihood instead of a Gaussian likelihood. The Cauchy distribution is a unimodal distribution with fatter tails than a Gaussian. Posterior distributions show the probability that OV cells respond with any average firing rate to each feature. The posterior distributions are shown for the dark gray contrast (left), the gray contrast (middle) and the white contrast (right). The results are similar to the results in Fig. $5 \mathrm{~d}$ and e. The main difference is that here the responses to the dark gray and gray contrasts also have a high probability of being above $0 \mathrm{~Hz}$. In contrast, with the Gaussian likelihood, there was a nonnegligible amount of probability mass below $0 \mathrm{~Hz}$ for both the dark gray and gray contrasts (Fig. $5 \mathrm{~d}$ and e). Thus, the overall pattern of results is the same (or stronger) when using a Cauchy distribution and confirms that our conclusions are robust to using likelihood functions with widely different tails (fat tails in the case of the Cauchy distribution; light tails in the case of the Gaussian distribution). 